medRxiv preprint doi: https://doi.org/10.1101/2020.07.31.20166090; this version posted August 4, 2020. The copyright holder for this preprint (which was not certified by peer review) is the author/funder, who has granted medRxiv a license to display the preprint in It is made available under a CC-BY-NC-ND 4.0 International license.

\title{
$3 \quad$ Land Use Change and Coronavirus Emergence Risk
}

4 Maria Cristina Rulli ${ }^{*}$, Paolo D'Odorico ${ }^{2 *}$, Nikolas Galli ${ }^{1}$, and David T.S. Hayman ${ }^{3}$

$5 \quad{ }^{1}$ Department of Civil and Environmental Engineering, Politecnico di Milano, Piazza Leonardo da

6 Vinci, 20133 Milano, Italy.

$7 \quad 2$ Department of Environmental Science, Policy, and Management, University of California,

8 Berkeley, California, USA.

$9{ }^{3}$ Molecular Epidemiology and Public Health Laboratory, School of Veterinary Science, Massey

10 University, New Zealand.

11 * Maria Cristina Rulli Email: mariacristina.rulli@polimi.it

12 * Paolo D’Odorico Email: paolododo@berkeley.edu

14 Sentence summarizing manuscript

15 Wildlife reservoirs for SARS-coronavirus-2 live in global hotspots of forest fragmentation, livestock, and 16 human density in China

17 Classification

18 Applied Ecology

19 ENVIRONMENTAL STUDIES

20 Keywords

21 COVID19, Land Use Change, One Health, Livestock, Wildlife

22

23 This PDF file includes:

$24 \quad$ Main text

25 Figures 1 to 4

26 Supporting information (SI) Tables 1 to 3, Figure 1 to 8

27 


\section{Abstract}

Coronavirus disease 2019 (COVID-19) and severe acute respiratory syndrome (SARS) causing coronaviruses are mostly discovered in Asian horseshoe bats. It is still unclear how ongoing land use changes may facilitate SARS-related coronavirus transmission to humans. Here we use a multivariate hotspot analysis of high-resolution land-use data to show that regions of China populated by horseshoe bats are hotspots of forest fragmentation, livestock and human density. We also identify areas susceptible to new hotspot emergence in response to moderate expansion of urbanization, livestock production, or forest disturbance, thereby highlighting regions vulnerable to SARS-CoV spillover under future land-use change. In China population growth and increasing meat consumption associated with urbanization and economic development have expanded the footprint of agriculture, leading to human encroachment in wildlife habitat and increased livestock density in areas adjacent to fragmented forests. The reduced distance between horseshoe-bats and humans elevates the risk for SARS-related coronavirus transmission to humans.

\section{Introduction}

The ongoing severe acute respiratory syndrome coronavirus-2 (SARS-CoV-2) pandemic is claiming human lives and disrupting the functioning of human societies in unprecedented ways. Where did this virus come from and how did it transmit to humans? What facilitated such a host shift and how can we prevent this from happening again? These are important questions, in addition to the most outstanding ones about whether, when, and how this pandemic will end.

Recent years have seen a rise in the recorded number of epidemics from emerging diseases. Such epidemics constitute a major public health threat because of our limited knowledge of prevention and treatment therapies. Most emerging infectious diseases originate from pathogen spillovers from wildlife to humans (1). Why are such outbreaks increasing? Does environmental change play a role?

Crucial to understanding the emergence of infectious diseases is the analysis of the factors facilitating the spillover from wildlife prior to further spread within human populations $(2,3)$. In the case of SARS-CoV-2 genomic sequencing has shown that the virus is most closely related ( 96\%) to a strain present in horseshoe bats (4). It is still unclear whether the spillover of SARS$\mathrm{CoV}-2$ occurred directly from bats to humans or through an intermediate species. For instance, a strain of coronavirus very similar to SARS-CoV-2 was detected in Malayan Pangolin (Manis javanica) (5), a wild mammal that is frequently illegally smuggled from Southeast Asia into China and sold in markets (5). Regardless of the specific pathway, the pathogen flow of emerging zoonotic diseases to humans is the result of human interactions with wildlife. We argue that the increasing incidence of emerging disease outbreaks is the result of a similar set of drivers able to change the distance and contact rates between wildlife and humans (as well as human-human interaction), including population growth, urbanization, increasing affluence in mid-income countries, and the associated dietary shifts (6). As countries become more affluent demand for animal products increases, leading to an expansion of agriculture and animal husbandry, often at the expense of natural ecosystems (7). Human penetration in wildlife habitat favors the interaction between humans and wildlife species, either directly through activities like hunting or through other species, particularly livestock that are in closer contact with humans (8-11). The

70 establishment of pastures, plantations or concentrated livestock farms close to forest margins 71 may increase pathogen flows from wildlife to humans $(2,9,11-14)$. Indeed, deforestation and 
72 forest fragmentation themselves reshape the dynamics of wildlife communities, possibly leading to the extinction of habitat specialist species, while allowing generalists to thrive (15).

While several reports in the media have conjectured on a possible link between land use change and the emergence of the COVID19 pandemic, such a hypothesis still has to be supported by a comprehensive high-resolution analysis of land use patterns that combines forest fragmentation to livestock and human encroachment in wildlife habitat (2). Here we analyze environmental changes to explain why China remains at risk for other SARS-related coronavirus outbreaks $(1,2)$. We analyze a set of factors that could make China a suitable location for the spillover to humans to occur. To that end, while we do not specifically link environmental change or bats as the immediate hosts of the SARS-CoV-2 ancestor $(4,5)$, we use horseshoe bats in the genus Rhinolophus (family Rhinolophidae) as a model system to understand the risk of future coronavirus outbreaks because China is reported to be a region with both highly diverse horseshoe bats and bat SARS-like CoV (16-18).

\section{Results}

Among the four CoV genera, two (alpha- and betacoronaviruses) are found in bats. Among the betacoronaviruses, all four subgenera have been discovered in bats, including the SARS-related CoVs (SARSr-CoV, subgenera Sarbecovirus) (16-18). Both SARS-CoV-1 and Swine acute diarrhoea syndrome coronavirus (SADS-CoV) emerged in southeast China and were later detected in horseshoe bats, mainly Rhinolophus sinicus and Rhinolophus affinis (4,16-18). Most SARSr-CoVs are detected in horseshoe bats, although some strains have been detected in other genera. SARSrCoVs in China are most similar to the highly pathogenic human SARS-CoVs $(4,16-18)$. Therefore, we performed our local analyses of disturbance at bat locations to horseshoe bats in China (Fig 1a; Table S1) and our analyses of disturbance within horseshoe bat distributions in both the larger South, East, and South East Asia region and then China.

Within these distributions we generated 10000 random sampling points (Fig 1a, see SI). Within $30 \mathrm{~km}$ from every random sampling point we calculate livestock density $\left(\mathrm{n} / \mathrm{km}^{2}\right)$, forest cover and fragmentation, cropland cover, population density, and the fractional cover of human settlements (see Supplementary Materials) (14). Hotspots were calculated (Fig 1a) using the Getis-Ord algorithm to show the areas with high or low values of land use attributes cluster (Fig 2).

China exhibits a relatively high concentration of livestock production in horseshoe bat distributions (Fig 1a, Table S1). Indeed, China is a hotspot of livestock density within this region (Fig 1b), with statistically significant higher concentrations of chickens, ducks, pigs, goats and cattle (Fig 2a). Within a $30 \mathrm{~km}$ radius from observed bat locations the density of chicken, ducks, pigs, goats, and cattle was again significantly greater than randomly selected locations. Conversely the sheep density is lower in China, though sheep density was low overall, as it was for other ruminants. The density of chickens, pigs, goats and cattle surrounding $(<30 \mathrm{~km})$ the points where these bats were recorded and at the randomly selected locations in China within the suitability region were not significantly different, indicating that these random locations have livestock densities that are representative of the areas in which the actual presence of horseshoe bats has been documented.

Forest cover and fragmentation have been related to virus outbreaks from wildlife (including bats) for other zoonotic diseases such as Ebola virus disease (14). China exhibits on average lower forest cover and cropland density and greater forest fragmentation than the other regions analyzed (Fig 
1c). The average forest cover and forest fragmentation in the surroundings (within $30 \mathrm{~km}$ distance) of random points selected in China and the other regions (Figs 1a, 2b) show that these differences are statistically significant. Likewise, statistically significant differences (i.e. lower average cover and higher average fragmentation) are found between the points of actual observations of horseshoe bats and randomly selected locations in the regions outside China within the distributions of these bats (Fig $2 b$ ).

China also exhibits higher levels of human presence in horseshoe bat distributions, as evidenced by population density and the fraction of the landscape covered by villages, towns, and other human settlement (Fig 2c). Indeed, the region of China suitable for horseshoe bats coincides with hotspots of human settlements (Fig 1d). Collectively, these results demonstrate that China exhibits stronger signs of human encroachment, livestock density, and forest disturbance of SARSr-CoV hosting horseshoe bat distributions than other regions. In China, regions close to forest fragments are more densely used for livestock production and human settlements - and consequently exhibit lower forest and cropland cover (Fig 1b) - thereby favoring the contact between wildlife and humans either directly or through intermediate animals such as livestock. The fact that China is a global hotspot in the concurrence of these three factors (fragmentation, livestock density, and human settlement) is highlighted by the multivariate hotspot analysis (Fig. 3). These three attributes account for bat habitat (fragmentation), livestock, and human presence, which are major factors contributing to the spillover of zoonotic infectious diseases. Interestingly, we find that China is the global hotspot of simultaneously high forest fragmentation, livestock density and human settlement. The other major global hotspots outside China are found in Java, Bhutan, east Nepal, northern Bangladesh, the Kerala state of India and North-East India, the latter of which are known for past outbreaks of Nipah virus, another bat-related zoonotic disease (19).

We then use the multivariate hotspot framework to identify regions at high potential risk of SARSr-CoVs spillovers to humans as a result of land use change. To that end the results of the multivariate hotspot analysis were clustered in 30 groups, based on geographic contiguity and similarity in the above three attributes (Fig. 4a; Table S3). We then perturbed one attribute at a time in each group to evaluate that group's susceptibility to transitioning from non-significant conditions (Fig. 3) to a hotspot state (Fig. 4b). This sensitivity analysis (Fig 4b) shows areas at risk of transitioning to hotspots as a result of a future increase in at least one of the analyzed attributes (i.e. forest fragmentation, livestock density, or human settlement). Interestingly the Chinese region south of Shanghai is at high risk of potentially turning into a hotspot as a result of fragmentation increase. Other regions susceptible to hotspot transition as a result of forest fragmentation include Japan and north Philippines. Likewise, the transition region between China's hotspot and Indochina's coldspot and the region surrounding the hotspot of Thailand could turn into hotspots for SARSr-CoV spillover as a result of increasing presence of livestock or humans, respectively (Fig. 4b). These results point both to regions of the world currently suitable for SARSr-CoV spillover from wildlife to humans as well as those at risk of becoming prone to spillover as a result of trajectories of land use change and human penetration (Fig. 4c; Fig.S8 SI)

\section{Discussion}

156 Our approach uses the horseshoe bats as a model family because of their key role as hosts of 157 Sarbecovirus coronaviruses, which have caused SARS, COVID-19, and SADS (4,13-18). Other 
strains of related viruses have been found in other bat genera, but these relationships are less clear (16-18). The widespread sampling of other bats may find species-specific relationships, though horseshoe bats appear to be the reservoirs where most SARSr-CoVs have their evolutionary ancestors so we assume they are the most appropriate model. The risk to humans from other coronaviruses, therefore, will be different, because their host distributions are different, and two CoV genera (gamma- and delta-coronaviruses) are mostly bird viruses.

164 The bat location data and species distribution data also suffer from different, but related issues. The bat location data are presence only data. True absence data are difficult to obtain, therefore we randomly sampled within different locations to generate pseudo-absence data. Choosing where to sample from also present difficulties, so we chose horseshoe bat distribution data for species that existed within China, East Asia, South, and South East Asia. This presents further issues as the distribution of one species, the greater horseshoe bat (Rhinolophus ferrumequinum), extends from Western Europe, Northern Africa, Central Asia and Eastern Asia. We therefore weighted our sampling based on the number of overlapping species distributions to account for this. However, these species distributions are large polygons and the realized niches used within them by the species likely differ, so better niche models using presence and, ideally, presence/absence data are required to develop better species presence predictions (20).

175 However, our results for random locations in China and outside China and reported bat 176 observations were comparable.

177 More generally, though using the relatively specific bat and virus relationships, we took a high-

178 level approach to understand the more distal or ultimate (rather than proximal) causes of 179 infectious disease emergence in China, linking environmental change and human drivers like 180 agricultural intensification. Different infectious diseases have different transmission mechanisms 181 and life cycles, and not all will respond to such changes in the same way. For example, directly 182 transmitted, acute infections with short incubation and infectious periods, like SARSr-CoVs, will 183 likely be dependent on hosts having greater densities, like in China, for them to emerge. The 184 epidemic potential is then also increased through local and global movement and trade, either of 185 people, wildlife, or livestock (8,21-23). Along with the biological properties of the virus and hosts, 186 the true risk of both the initial cross-species transmission and epidemic potential is either 187 increased or limited by more proximal mechanisms, such as biosecurity, health and safety 188 measures (e.g. personal protective equipment, meat hygiene) that can reduce risk, even if the 189 ultimate factors are present and increasing through the processes of habitat fragmentation and 190 human encroachment $(8,14)$.

191 Spillover of infectious disease such as SARS, COVID-19 and SADS from wildlife to humans likely 192 requires the coexistence of horseshoe bats and humans in the same environment and is favored 193 by the presence of intermediate animal species, particularly livestock because it is in closer 194 contact with humans. The fragmentation and disturbance of forest ecosystems likely favors 195 habitat generalist bat species. In particular, chickens, ducks, and pigs have been associated with 196 the spread of several zoonotic viral infections, such as influenza viruses. This study demonstrates 197 that in China these important factors responsible for reducing the distance between wildlife and 198 humans co-occur both in horseshoe bat distributions and in the surroundings of actual 199 documented bat occurrence. These results are consistent with the notion that population growth 200 and increasing meat consumption associated with urbanization and economic growth have 201 expanded the footprint of agriculture, leading to human encroachment in wildlife habitat and 202 increased livestock density in areas adjacent to fragmented forest patches. China has dramatically 
increased animal consumption (24), likely as the result of increasing affluence. In China, meat supply is largely reliant on domestic production using imported feed (e.g. soy from the Americas) (24), which explains the high livestock density in many rural areas, including those at the forest margins. Likewise, economic growth and the shift to diets richer in animal products explains the increasing demand for wild animal meat delicacies, increasing human-wildlife interactions through multiple pathways and the disturbance of forest habitat in more remote locations frequently abroad - through trade-related teleconnections (25).

210

211

212

213

214

215

216

217

218

219

220

221

222

223

224

225

226

227

228

229

230

231

232

233

234

235

236

237

238

239

240

241

242

The multivariate hotspot analysis highlights how China is the largest hotspot for the concurrence of high forest fragmentation, livestock density, and human presence in our analysis (Fig. 3). The sensitivity analyses identifying the possible transition to new hotspots in response to an increase in one of these attributes (Fig. 4b) highlights areas that could become suitable for spillover and the type of land use change that could induce hotspot activation. Therefore, this analysis highlights region-specific targeted interventions that are urgently needed to increase resilience to SARSr-CoV spillovers. For instance, the green dots in Fig. 4 could be turned into hotspots as a result of forest fragmentation. In these regions resilience can be built through forest conservation or restoration efforts. Indeed, land use change evaluations should consider the risk of activating new hotspots suitable for wildlife-to-human spillover of pathogens such as SARSr-CoV, an aspect that has seldom been included in the impact analysis of land use change. Likewise, other regions such as the China-Indochina transition zone or central Thailand are prone to hotspot transitioning as a result of increased livestock density of urbanization, respectively. In these cases, mitigation of SARSr-CoV emergence can been enhanced by reducing livestock or human density, respectively, thereby inverting ongoing dietary and urbanization trends. Thus, environmental health, is tightly connected to both animal and human health, as recently stressed by planetary and 'one health' discourses, which advocate for more holistic views of global health, encompassing environment, animals, and people as well as their interactions (26).

\section{Materials and Methods}

\section{Bat location data}

Most SARS-related CoVs are detected in horseshoe bats, although some strains have also been detected in other genera (22,27-33). SARSr-CoVs in China are most similar to the highly pathogenic human SARS-CoVs (34-36).

We restricted our local analyses of disturbance at bat locations to Rhinolophid bats in China. We performed a Web of Science ${ }^{\circledR}$ search on 10/04/2020 using the following Boolean Operators: Rhinoloph* AND China AND Monitor* OR Survey OR Niche OR Distribution. We found 129 unique references. We removed all those published before 2000, reporting data outside China, review articles, and non-English (specifically 23 Chinese language publication), those with no Rhinolophid data, and those reporting only fossil records. We kept infection studies. This left 48 publications. We then further manually reviewed the publications for those reporting location data (22) but more specifically those with latitude and longitude, leaving 17 publications and 264 observations (see Fig. 1a; Table S1).

\section{Bat distribution data}

We restricted our analyses of disturbance in bat distributions to Rhinolophid bats in both the larger South, East and South East Asia region (but see main manuscript) and then China. We searched the IUCN Red List database (https://www.iucnredlist.org/search) using Taxonomy: 
Rhinolophidae and Region: East Asia and South \& South East Asia (herein 'regional') followed by Taxonomy: Rhinolophidae and Region: East Asia: China, Hong Kong \& Taiwan (herein 'Chinese') classifications and downloaded the shapefiles for the 55 regional and 22 Chinese Rhinolophus species present in the region. We consider these areas as regions of suitable habitat for

251 Rhinolophidae. The extent of this study area exceeds 28.5 million $\mathrm{km}^{2}$.

252 Within these putative species distributions, we generated 10000 random sampling points with a local sampling density that is proportional to the number of species whose distributions were reported at the point. For every random sampling point we consider a circular area of $30 \mathrm{~km}$ radius within which we calculate livestock density, forest cover and fragmentation, cropland cover, population density and the fractional cover of human settlements as explained below. The average values of these statistics are then calculated for China and the other regions of the world with habitat suitable for Rhinolophidae and compared and the difference is tested for significance using the Mann-Whitney non-parametric test in Mathematica $\bigodot$.

\section{Livestock, forest cover, and population data}

261 We took livestock data from the GeoWiki database that provides georeferenced livestock counts (in heads $/ \mathrm{km}^{2}$ ) at $1 \mathrm{~km}$ resolution for chickens, ducks, pigs, goats, sheep, and cattle (https://livestock.geo-wiki.org/home-2/). We quantified human presence both in terms of population density at $1 \mathrm{~km}$ resolution and as fraction of the landscape taken by villages, towns or other settlements from the WorldPop database at $1 \mathrm{~km}$ resolution. We used cropland data (at $30 \times 30 \mathrm{~m}^{2}$ resolution) from (37). Forest cover data are available at $30 \mathrm{~m}$ resolution annually between 2000 and 2018 (38). Forest cover is associated with the presence of trees taller than 5 $\mathrm{m}$. Forest loss or gain was determined as the difference in forest cover between these two years.

\section{Forest fragmentation analyses}

270 We performed a forest fragmentation analyses based on Vogt et al. (39) using the $30 \mathrm{~m}$ forest 271 cover data. This method distinguishes forest cores, from forest margins and patches. Every $30 \mathrm{~m}$ 272 pixel is classified as wooded or non-wooded, based on whether its tree cover was greater or 273 smaller than $50 \%$ in the year 2018. Forest cores are wooded pixels that are not adjacent to non274 wooded pixels. Conversely, forest patches are made of wooded pixels that are not adjacent to 275 forest core pixels. Wooded pixels that are neither core nor patch pixels occur at the margins of 276 forest cores. Forest fragmentation was then quantified in terms of a composite fragmentation 277 index (CFI) (13), defined as the ratio between the sum of number of pixels classified as "margins", 278 "patches", or smaller core areas (i.e., $<200 \mathrm{ha}$ ), and the total number of pixels (wooded + non279 wooded) in the $30 \mathrm{~km}$ circles used to characterize land cover and land use in the surroundings of 280 the points of actual bat observations or the randomly generated points. This index ranges 281 between 0 and 1.

\section{Hotspot Analyses and multivariate clustering}

283 We mapped statistically significant hotspots of livestock density, forest fragmentation, and 284 human settlements. To be a hotspot, an area needs to have a high (or low, in the case of cold285 spots) value of its attribute and be also surrounded by other areas with high (or low) values of the 286 same attribute. To that end, the $30 \mathrm{~km}$ circles centered on the 10000 random points (Fig. 1a) 287 considered in this study were used to carry out a hotspot analysis applying the Getis-Ord 288 algorithm (Gi* statistics). The $\mathrm{Gi}^{*}$ statistic shows to what extent areas with high or low values tend 
medRxiv preprint doi: https://doi.org/10.1101/2020.07.31.20166090; this version posted August 4, 2020. The copyright holder for this preprint (which was not certified by peer review) is the author/funder, who has granted medRxiv a license to display the preprint in

It is made available under a CC-BY-NC-ND 4.0 International license.

289 to cluster thereby forming a hotspot. The result of the $\mathrm{Gi}^{*}$ analysis is reported in terms of

290 statistically significance with $90 \%, 95 \%$, and $99 \%$ confidence (Fig. 1).

291 We then used two different methods to generate a multivariate distribution for the three 292 indicators (livestock density, forest fragmentation and human settlements). First, we averaged 293 their $\mathrm{Gi}^{*}$. Since the $\mathrm{Gi}^{*}$ is a $\mathrm{z}$-score, i.e. it has a standard normal distribution, a linear combination 294 of the three $\mathrm{Gi}^{*}$ indicators, such as their average, is a standard normal distribution and can still 295 be represented with the same significance levels (Fig. 2). Second, we performed a spatially 296 constrained multivariate clustering analysis. A Minimum Spanning Tree (MST) from the 297 connectivity graph of the features was built, and then the SKATER (Spatial "K"luster Analysis by 298 Tree Edge Removal) clustering method was used (40). The SKATER iteratively cuts branches in the 299 MST, based on data variability among and within groups and on a spatial constraint, until it 300 reaches the user-defined number of groups. The spatial constraint defined here is a $k$ nearest

301

302

303

304

305

306

307

308

309

310

311

312

313

314

315

316

317

318

319

320 neighbors type with 8 neighbors, meaning each feature in a group must have at least one of its 8 nearest neighbors in the same group. We chose 30 as the number of groups, calculated a set of summary statistics and boxplots for the groups and compared them to their global values (Table S2). For each indicator, we calculated the $R^{2}$ value as the reduction in variance of the indicator obtained by grouping, divided by the original variance of the indicator (Table S2). While the modularity analysis based on pseudo F-statistics shows that the optimal number of groups (the maximum differences between groups while maximizing within group similarity) is 12 , here we studied 30 groups to analyse distinct regional patterns. Having a greater number of groups allows us to identify groups that are susceptible to transitioning to a hotspot because they are not "too different" from hotspots.

\section{Acknowledgments}

M.C.R and N.G. are supported by Cariplo Foundation (SusFeed project 0737 CUP D49H170000300007). D.T.H. is supported by USDA Hatch Multistate project no. W4190 capacity fund; Rutherford Discovery Fellowship RDF-MAU1701.

Competing interest statement: The authors declare no competing interests.

Author contributions: M.C.R. designed research; M.C.R. and D.T.S.H performed research; M.C.R., P.D. and N.G. analyzed data; P.D., M.C.R. and D.T.S.H wrote the paper. 


\section{References}

1 K. E. Jones, N. G. Patel, M. A. Levy, A. Storeygard, D. Balk, J. L. Gittleman, P. Daszak, Global trends in emerging infectious diseases. Nature 451, 990-993 (2008).

2 T. Allen, K. Murray, C. Zambrana-Torrelio, S. S. Morse, C. Rondinini, M. Di Marco, N. Brerit, K. J. Olival, P. Daszak, Global hotspots and correlates of emerging zoonotic diseases, Nature Coomunications, 8: 1124, DOI: 10.1038/s41467-017-00923-8 (2017).

3 T. Wu C. Perrings, A. Kinzig, J. P. Collins, B. A. Minteer, P. Daszak, Economic growth, urbanization, globalization, and the risks of emerging infectious diseases in China: $A$ review, Ambio, 46:18-29.

$4 \quad$ Peng Zhou, Xing-Lou Yang, Xian-Guang Wang, Ben Hu, Lei Zhang, Wei Zhang, Hao-Rui Si, Yan Zhu, Bei Li, Chao-Lin Huang, Hui-Dong Chen, Jing Chen, Yun Luo, Hua Guo, Ren-Di Jiang, Mei-Qin Liu, Ying Chen, Xu-Rui Shen, Xi Wang, Xiao-Shuang Zheng, Kai Zhao, QuanJiao Chen, Fei Deng, Lin-Lin Liu, Bing Yan, Fa-Xian Zhan, Yan-Yi Wang, Geng-Fu Xiao \& Zheng-Li Shi, A pneumonia outbreak associated with a new coronavirus of probable bat origin. Nature 579, 270-273, (2020).

5 Tommy Tsan-Yuk Lam, Na Jia, Ya-Wei Zhang, Marcus Ho-Hin Shum, Jia-Fu Jiang, Hua-Chen Zhu, Yi-Gang Tong, Yong-Xia Shi, Xue-Bing Ni, Yun-Shi Liao, Wen-Juan Li, Bao-Gui Jiang, Wei Wei, Ting-Ting Yuan, Kui Zheng, Xiao-Ming Cui, Jie Li, Guang-Qian Pei, Xin Qiang, William Yiu-Man Cheung, Lian-Feng Li, Fang-Fang Sun, Si Qin, Ji-Cheng Huang, Gabriel M. Leung, Edward C. Holmes, Yan-Ling Hu, Yi Guan \& Wu-Chun Cao, Identifying SARS-CoV-2 related coronaviruses in Malayan pangolins. Nature, (2020).

6 D. Tilman \& M. Clark, Global diets link environmental sustainability and human health. Nature 515, 518-522 (2014).

7 R. Naylor, Expanding the boundaries of agricultural development. Food Security 3, 233 (2011).

8 D. A. Wilkinson, J. C. Marshall, N. P. French, D. T. Hayman, Habitat fragmentation, biodiversity loss and the risk of novel infectious disease emergence. Journal of the Royal Society Interface 15, 20180403 (2018).

9 C. K. Johnson, P. L. Hitchens, P. S. Pandit, J. Rushmore, T. S. Evans, C. C. W. Young, M. M. Doyle, Global shifts in mammalian population trends reveal key predictors of virus spillover risk. Proceedings of the Royal Society B 287 (2020).

10 L. S. P. Bloomfield, T. L. McIntosh, E. F. Lambin, Habitat fragmentation, livelihood behaviors, and contact between people and nonhuman primates in Africa. Landscape Ecology 35, 985-1000, doi:10.1007/s10980-020-00995-w (2020).

11 J. R. C. Pulliam, J. H. Epstein, J. Dushoff, S. A. Rahman, M. Bunning, A. A. Jamaluddin, A. D. Hyatt, H. E. Field, A. P. Dobson, P. Daszak, the Henipavirus Ecology Research Group (HERG), Agricultural intensification, priming for persistence and the emergence of Nipah virus: a lethal bat-borne zoonosis. Journal of the Royal Society Interface 9, 89-101 (2012).

12 K. B. Chua, K. J. Goh, K. T. Wong, A. Kamarulzaman, P. S. Tan, T. G. Ksiazek, S. R. Zaki, G. Paul, S. K. Lam, C. T. Tan, Fatal encephalitis due to Nipah virus among pig-farmers in Malaysia. The Lancet 354, 1257-1259 (1999).

13 Peng Zhou, Hang Fan, Tian Lan, Xing-Lou Yang, Wei-Feng Shi, Wei Zhang, Yan Zhu, Ya-Wei Zhang, Qing-Mei Xie, Shailendra Mani, Xiao-Shuang Zheng, Bei Li, Jin-Man Li, Hua Guo, Guang-Qian Pei, Xiao-Ping An, Jun-Wei Chen, Ling Zhou, Kai-Jie Mai, Zi-Xian Wu, Di Li, Danielle E. Anderson, Li-Biao Zhang, Shi-Yue Li, Zhi-Qiang Mi, Tong-Tong He, Feng Cong, Peng-Ju Guo, Ren Huang, Yun Luo, Xiang-Ling Liu, Jing Chen, Yong Huang, Qiang Sun, 
Xiang-Li-Lan Zhang, Yuan-Yuan Wang, Shao-Zhen Xing, Yan-Shan Chen, Yuan Sun, Juan Li, Peter Daszak, Lin-Fa Wang, Zheng-Li Shi, Yi-Gang Tong, Jing-Yun, Fatal swine acute 255-258(2018).

M. C. Rulli, M. Santini, D. T. Hayman, P. D'Odorico, The nexus between forest fragmentation in Africa and Ebola virus disease outbreaks. Scientific reports 7, 41613 (2017). in a changing world 63-103 (Springer, Cham, 2016). Microbiology 17, 181-192(2019). Viruses 11, doi:10.3390/v11020174 (2019).

19 V. Soman Pillai, G. Krishna, M. Valiya Veettil, Nipah Virus: Past Outbreaks and Future Containment. Viruses. 12(4):465 (2020).

20 W. Thuiller, B. Lafourcade, R. Engler, M. B. Araújo, BIOMOD-a platform for ensemble forecasting of species distributions. Ecography 32, 369-373 (2009).

21 M. Chinazzi, J. T. Davis, M. Ajelli, C. Giovannini, M. Litvinova, S. Merler, A. Pastore y Piontti, K. Mu, L. Rossi, K. Sun, C. Viboud, X. Xiong, H. Yu, M. E. Halloran, I. M. Longini Jr., A. Vespignani, The effect of travel restrictions on the spread of the 2019 novel coronavirus (COVID-19) outbreak. Science 368, 395-400 (2020).

22 M. U. G. Kramer, C. Yang, B. Gutierrez, C. Wu, B. Klein, D. M. Pigott, Open CoVID-19 Data Working Group, L. du Plessis, N. R. Faria, R. Li, W. P. Hanage, J. S. Brownstein, M. La, J. S. Brownstein, M. Layan, A. Vespignani, H. Tian, C. Dye, O. G. Pybus, S. V. Scarpino, The effect of human mobility and control measures on the COVID-19 epidemic in China. Science 368, 493-497 (2020).

23 Q. Yang, X. Zhao, P. Lemey, M. A. Suchard, Y. Bi, W. Shi, D. Liu, W. Qi, G. Zhang, N. C. Stenseth, G. Pybus, H. Tian, Assessing the role of live poultry trade in communitystructured transmission of avian influenza in China. Proceedings of the National Academy of Sciences 117, 5949-5954 (2020).

24 P. D'Odorico, K. F. Davis, L. Rosa, J. A. Carr, D. Chiarelli, J. Dell'Angelo, J. Gephart, G. K. MacDonald, D. A. Seekell, S. Suweis, M. C. Rulli, The global food-energy-water nexus. Reviews of Geophysics, 56, 456-531 (2018).

25 P. Meyfroidt, E. F. Lambin, K. H. Erb, T. W. Hertel, Globalization of land use: Distant drivers of land change and geographic displacement of land use. Current Opinion in Environmental Sustainability, 5(5), 438-444 (2013).

26 S. Whitmee, A. Haines, C. Beyrer, F. Boltz, A. G. Capon, B. Ferreira de Souza Dias, A. Ezeh, H. Frumkin, P. Gong, P. Head, R. Horton, G. M. Mace, R. Marten, S. S Myers, S. Nishtar, S. A. Osofsky, S. K. Pattanayak, M. J. Pongsiri, C. Romanelli, A. Soucat, J. Vega, D. Yach, Safeguarding human health in the Anthropocene epoch: report of The Rockefeller Foundation-Lancet Commission on planetary health. The Lancet 386, 1973-2028 (2015). P. C. Y. Woo, S. K. P. Lau, C. S. F. Lam, C. C. Y. Lau, A. K. L. Tsang, J. H. N. Lau, R. Bai, J. L. L. Teng, C. C. C. Tsang, M. Wang, B. J. Zheng, K. H. Chan, K. Y. Yuen, Discovery of seven novel Mammalian and avian coronaviruses in the genus deltacoronavirus supports bat coronaviruses as the gene source of alphacoronavirus and betacoronavirus and avian 
coronaviruses as the gene source of gammacoronavirus and deltacoronavirus. J. Virol., 86, 3995-4008, (2012). Drexler, Rooting the Phylogenetic Tree of Middle East Respiratory Syndrome Coronavirus by Characterization of a Conspecific Virus from an African Bat. J. Virol., 88, 11297-11303, (2014).

ICTV

2018:

Cornidovirineae.

Available

online: https://talk.ictvonline.org/taxonomy/p/taxonomy-history?taxnode id=20186105 (2018).

31 P. C. Woo, M. Wang, S. K. Lau, H. Xu, R. W. Poon, R. Guo, B. H. Wong, K. Gao, H. W. Tsoi, Y. Huang, K. S. Li, C. S. Lam, K. H. Chan, B. J. Zheng, K. Y. Yuen, Comparative analysis of twelve genomes of three novel group $2 \mathrm{c}$ and group $2 \mathrm{~d}$ coronaviruses reveals unique group and subgroup features. J. Virol., 81, 1574-1585, (2007).

C. Huang, W. J. Liu, W. Xu, T. Jin, Y. Zhao, J. Song, Y. Shi, W. Ji, H. Jia, Y. Zhou, H. Wen, H. Zhao, H. Liu, H. Li, Q. Wang, Y. Wu, L. Wang, D. Liu, G. Liu, H. Yu, E. C. Holmes, L. Lu, G. F. Gao, A Bat-Derived Putative Cross-Family Recombinant Coronavirus with a Reovirus Gene. PLoS Pathog, 12, e1005883 (2016). S. K. P. Lau, L. Zhang, H. K. H. Luk, L. Xiong, X. Peng, K. S. M. Li, X. He, P. S. Zhao, R. Y. Y. Fan, A. C. P. Wong, S.S. Ahmed, J.P. Cai, J. F. W. Chan, Y. Sun, D. Jin, H. Chen, T. C. K. Lau, R. K. H. Kok, W. Li, K. Y. Yuen, P. C. Y. Woo, Receptor Usage of a Novel Bat Lineage C Betacoronavirus Reveals Evolution of Middle East Respiratory Syndrome-Related Coronavirus Spike Proteins for Human Dipeptidyl Peptidase 4 Binding. J. Infect. Dis., 218, 197-207 (2018).

33 S. K. Lau, P. C. Woo, K. S. Li, Y. Huang, H. W. Tsoi, B. H. Wong, S. S. Wong, S. Y. Leung, K. H. Chan, K. Y. Yuen, Severe acute respiratory syndrome coronavirus-like virus in Chinese horseshoe bats. Proc. Natl. Acad. Sci. USA, 102, 14040-14045 (2005).

34 B. Hu, L. P. Zeng, X. L. Yang, X. Y. Ge, W. Zhang, B. Li, J. Z. Xie, X. R. Shen, Y. Z. Zhang, N. Wang, D. S. Luo, X. S. Zheng, M. N. Wang, P. Daszak, L. F. Wang, J. Cui, Z. L. Shi, Discovery of a rich gene pool of bat SARS-related coronaviruses provides new insights into the origin of SARS coronavirus. PLoS Pathog., 13, e1006698 (2017). K. G. Andersen, A. Rambaut, W. I. Lipkin, E. C. Holmes, R. F. Garry, The proximal origin of SARS-CoV-2. Nature medicine: 26(4):450-452 (2020). S. Lee, S. D. Jo, K. Son, I. An, J. Jeong, S. J. Wang, Y. Kim, W. Jheong, J. K. Oem, Genetic Characteristics of Coronaviruses from Korean Bats in 2016. Microb. Ecol., 75, 174-182, (2018).

P. Teluguntla, P. S. Thenkabail, J. Xiong, M. K. Gumma, C. Giri, C. Milesi, M. Ozdogan, R. Congalton, J. Tilton, T. R. Sankey, R. Massey, A. Phalke, K. Yadav, Global Cropland Area Database (GCAD) derived from Remote Sensing in Support of Food Security in the Twentyfirst Century: Current Achievements and Future Possibilities. Chapter 7, Vol. II. Land Resources: Monitoring, Modelling, and Mapping, Remote Sensing Handbook edited by Prasad S. Thenkabail (2014).

8 M. C. Hansen, P. V. Potapov, R. Moore, M. Hancher, S. Turubanova, A. Tyukavina, D. Thau, S. V. Stehman, S. J. Goetz, T. R. Loveland, A. Kommareddy, A. V. Egorov, L. Chini, C. O. Justice, J. R. G. Townshend, High-resolution global maps of 21st-century forest cover change. Science, 342:850-853 (2013). 
medRxiv preprint doi: https://doi.org/10.1101/2020.07.31.20166090; this version posted August 4, 2020. The copyright holder for this preprint (which was not certified by peer review) is the author/funder, who has granted medRxiv a license to display the preprint in It is made available under a CC-BY-NC-ND 4.0 International license.

$45839 \quad$ P. Vogt, K. H. Riitters, C. Estreguil, J. Kozak, T. G. Wade, J. D. Wickham, Mapping spatial patterns with morphological image processing. Landscape Ecol. 22, 171-177 (2007).

460 R. M. Assuncao, M. C. Neves, G. Camara, C. Da Costa Freitas, Efficient Regionalisation Techniques for Socio-economic Geographical Units using Minimum Spanning Trees. International Journal of Geographical Information Science 20 (7): 797-811 (2006). 
medRxiv preprint doi: https://doi.org/10.1101/2020.07.31.20166090; this version posted August 4, 2020. The copyright holder for this preprint (which was not certified by peer review) is the author/funder, who has granted medRxiv a license to display the preprint in perpetuity.

It is made available under a CC-BY-NC-ND 4.0 International license.

\section{Figures main text}

467

468
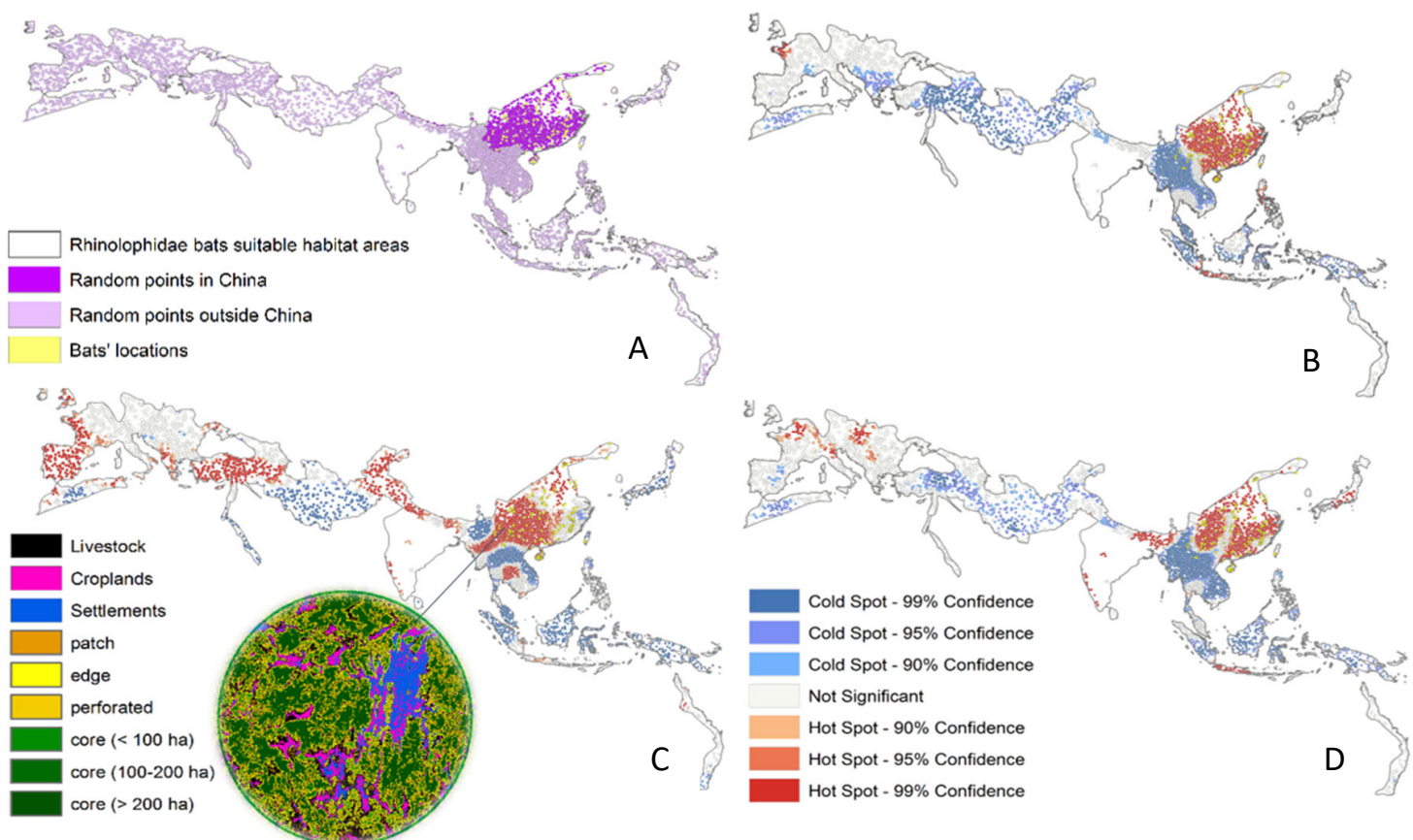

472 Figure 1. Univariate spatial analysis of coronavirus outbreak drivers (A) Sampling points

473 randomly generated within China (dark purple) and outside China (light purple) and bat location

474 points (yellow), weighted by the horseshoe bat species distributions present in East, South \&

475 South East Asia; (B) hotspots (red) and coldspots (blue) of livestock density; (C) hotspots of forest fragmentation; (D) hotspots of human settlement. 
medRxiv preprint doi: https://doi.org/10.1101/2020.07.31.20166090; this version posted August 4, 2020. The copyright holder for this preprint (which was not certified by peer review) is the author/funder, who has granted medRxiv a license to display the preprint in perpetuity.

It is made available under a CC-BY-NC-ND 4.0 International license .
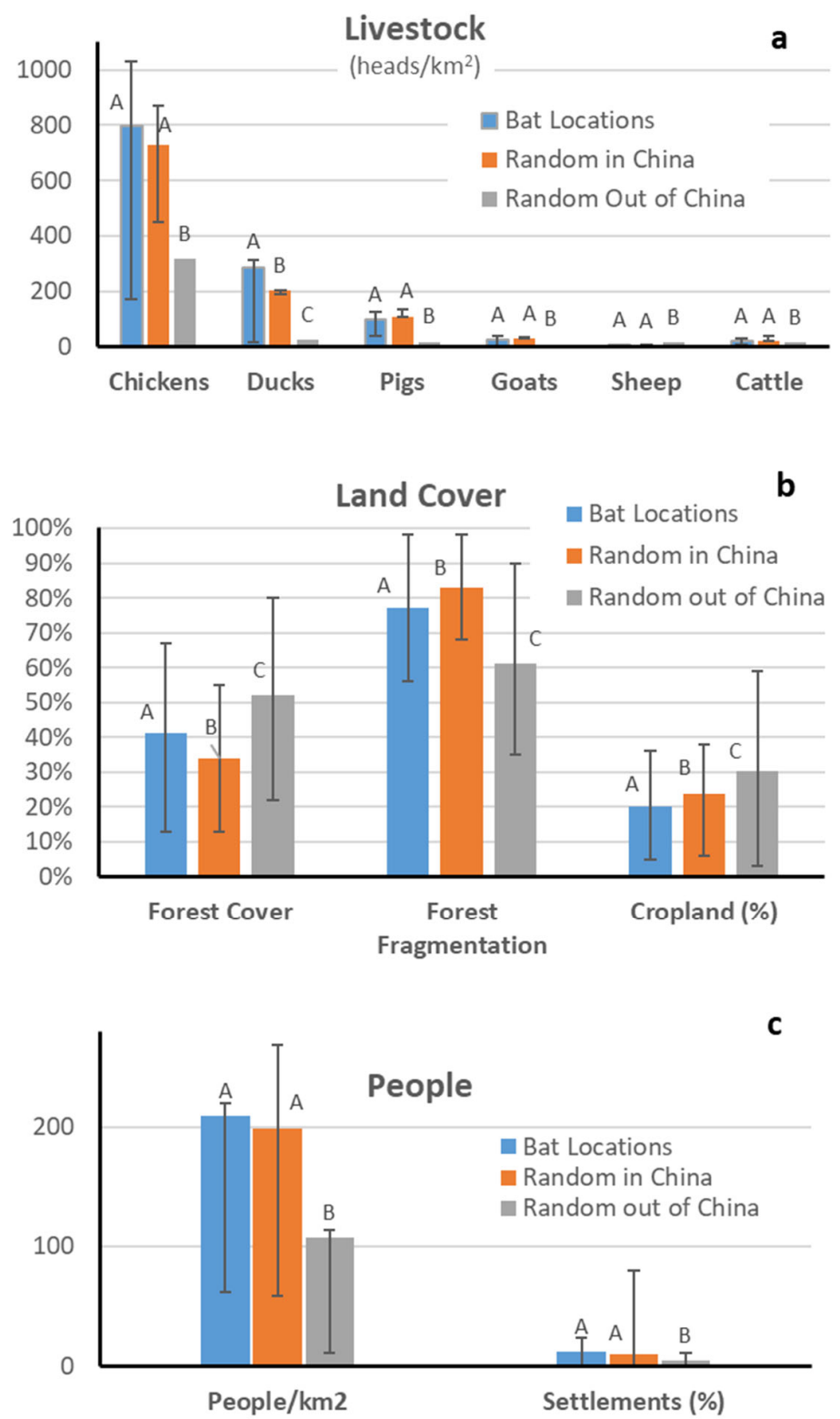

479 Figure 2. Distribution comparison for coronavirus outbreak drivers. Average distributions of 480 livestock (A), land cover and use (B), and human population (C) in areas likely suitable for 481 horseshoe bat occurrence in China and in the rest of their distribution. Error bars correspond to 482 the $20 \%$ and $80 \%$ percentiles. Different capital letters indicate statistically different means $483(\alpha=0.05)$. 
medRxiv preprint doi: https://doi.org/10.1101/2020.07.31.20166090; this version posted August 4, 2020. The copyright holder for this preprint (which was not certified by peer review) is the author/funder, who has granted medRxiv a license to display the preprint in It is made available under a CC-BY-NC-ND 4.0 International license.

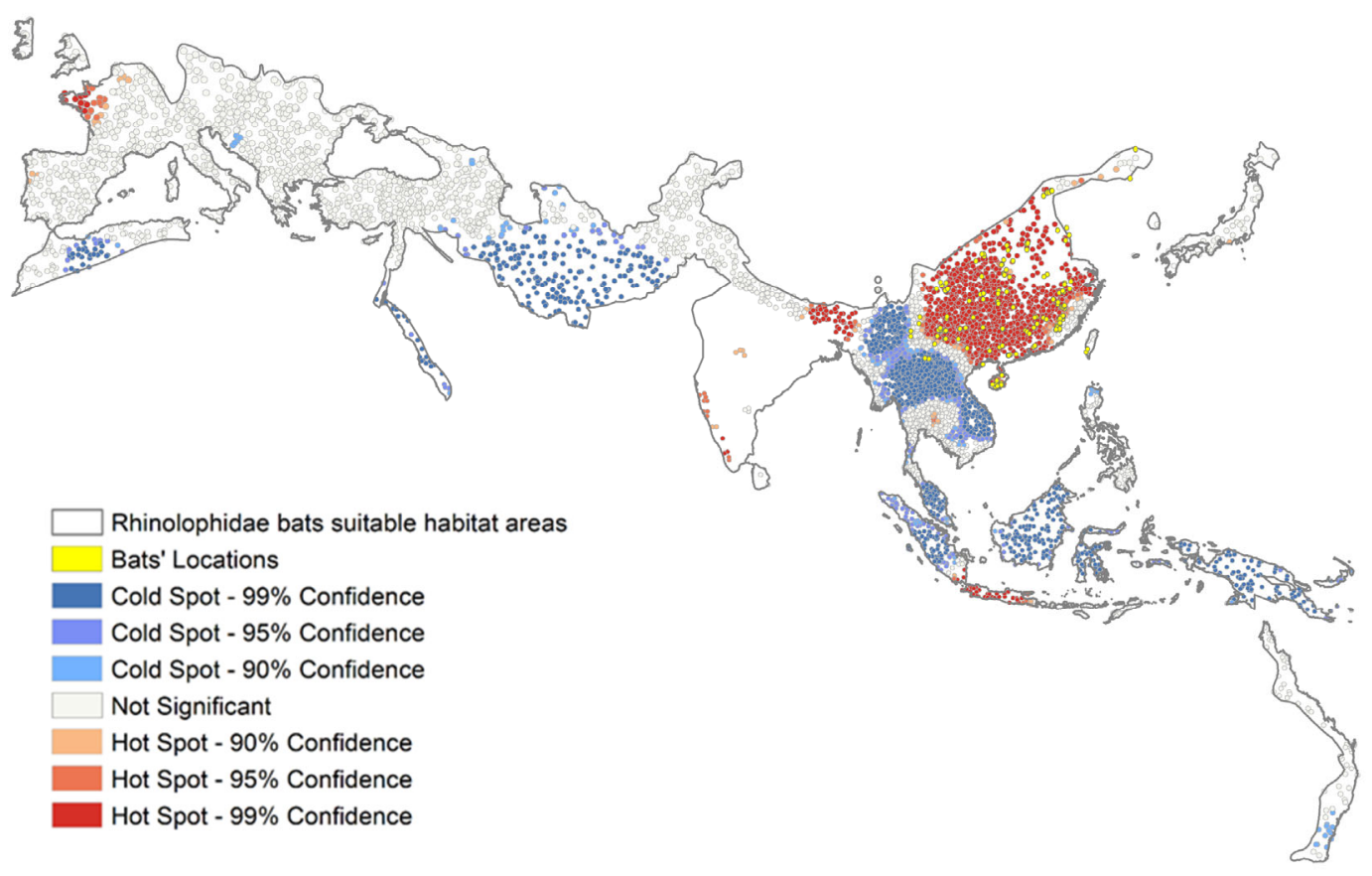

487 Figure 3. Multivariate spatial analysis of coronavirus outbreak drivers. Hotspot analysis based 488 on the average Gi* Z-Score values for fragmentation, livestock density, and human settlements. 489 Hotspots are classified based on their significance level. 
medRxiv preprint doi: https://doi.org/10.1101/2020.07.31.20166090; this version posted August 4, 2020. The copyright holder for this preprint (which was not certified by peer review) is the author/funder, who has granted medRxiv a license to display the preprint in It is made available under a CC-BY-NC-ND 4.0 International license.
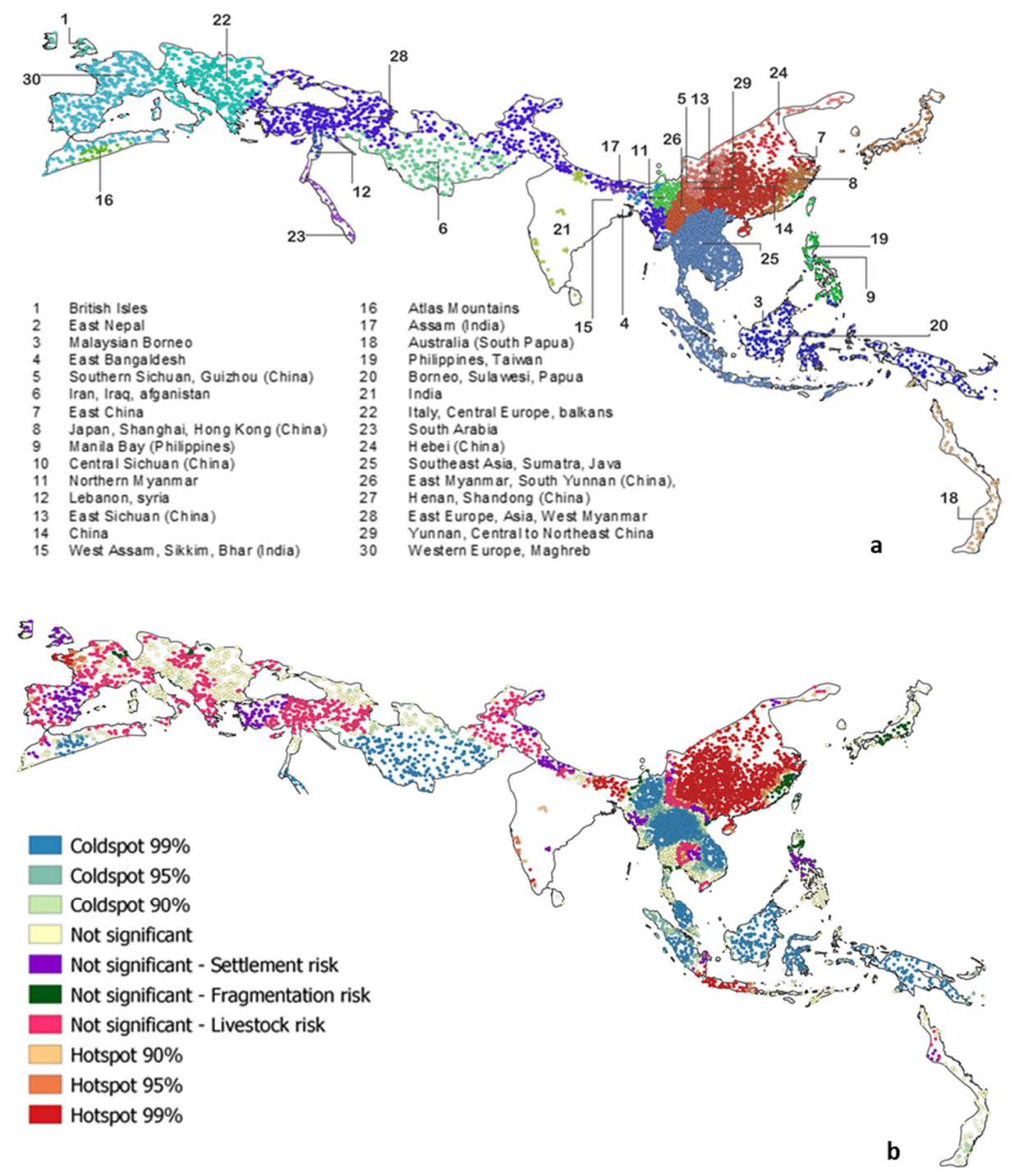

492
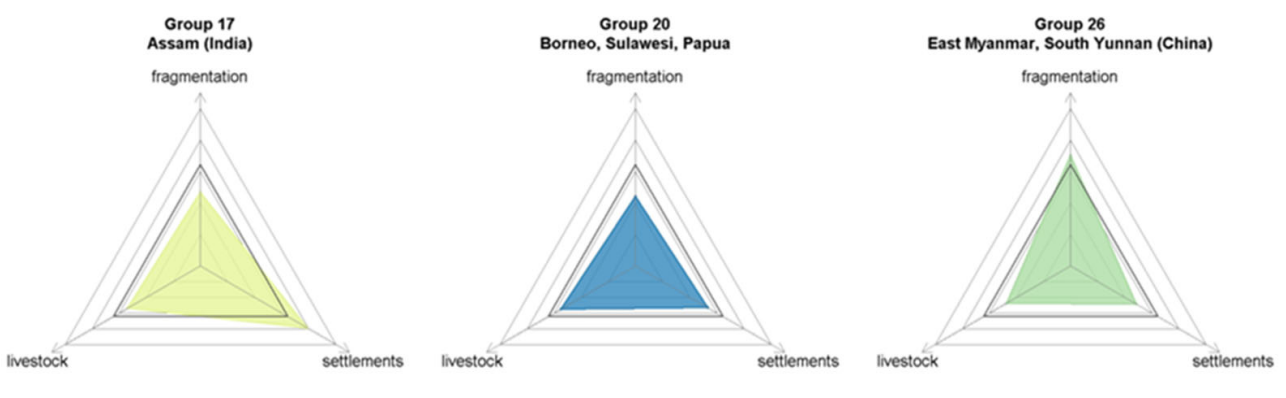

Figure 4. Multivariate grouping analysis of coronavirus spillover drivers. (A) Multivariate groups analysis based on fragmentation, livestock and settlements attributes; (B) Areas at risk of 
medRxiv preprint doi: https://doi.org/10.1101/2020.07.31.20166090; this version posted August 4, 2020. The copyright holder for this preprint (which was not certified by peer review) is the author/funder, who has granted medRxiv a license to display the preprint in perpetuity.

It is made available under a CC-BY-NC-ND 4.0 International license .

496 becoming hotspots as a result of changes in forest fragmentation (green), increase in livestock

497 density (fuchsia) and human settlement (purple); (C) Possible trajectories of hotspot transition.

498 These 3 groups represent areas not yet classified as hotspots (not significant by Gettis Ord

499 analysis), but which may change trajectory. The solid triangle represents the safe space of

500 variation for the 3 indicators (using multivariate Getis-Ord analysis).

501 
medRxiv preprint doi: https://doi.org/10.1101/2020.07.31.20166090; this version posted August 4, 2020. The copyright holder for this preprint (which was not certified by peer review) is the author/funder, who has granted medRxiv a license to display the preprint in

It is made available under a CC-BY-NC-ND 4.0 International license.

\section{Environmental Change and Coronavirus Emergence Risk}

504 Maria Cristina Rulli ${ }^{1}$, Paolo D'Odorico ${ }^{2}$, Nikolas Galli ${ }^{1}$, David T.S. Hayman ${ }^{3}$

$505{ }^{1}$ Department of Civil and Environmental Engineering, Politecnico di Milano, Piazza Leonardo da Vinci, 506 Milano, Italy

5072 Department of Environmental Science, Policy, and Management, University of California, Berkeley, 508 California, USA

$509{ }^{3}$ Molecular Epidemiology and Public Health Laboratory, School of Veterinary Science, Massey University, $510 \quad$ New Zealand

513 Figures and Tables Supplementary Materials (SI)

514 Table S1. Horseshoe bat locations based on literature survey of studies reporting occurrences and related coordinates (coordinates are here listed as reported in the original articles and therefore

516 they are not in a homogeneous format).

\begin{tabular}{|c|c|c|c|c|c|c|}
\hline Species & Site & Location & Region & Latitude & Longitude & Ref. \\
\hline R. macrotis & Shiyan Cave & $\begin{array}{l}\text { Jing gang shan Nature } \\
\text { Reserve }\end{array}$ & Jiangxi Province & $26^{\circ} 36^{\prime} \mathrm{N}$ & $114^{\circ} 12^{\prime} \mathrm{E}$ & 1 \\
\hline R. macrotis & Xianren Cave & Jinning County & Yunnan Province & $24^{\circ} 30^{\prime} \mathrm{N}$ & $102^{\circ} 20^{\prime} \mathrm{E}$ & \\
\hline R. macrotis & Tiantang Cave & $\begin{array}{l}\text { Changtang County, Nanning } \\
\text { city }\end{array}$ & Guangxi Province & $22^{\circ} 49^{\prime} \mathrm{N}$ & $108^{\circ} 42^{\prime} \mathrm{E}$ & \\
\hline R. luctus & Emei shan & Emei shan & Sichuan province & $29^{\circ} 31^{\prime} 11^{\prime \prime} \mathrm{N}$ & $103^{\circ} 19^{\prime} 57^{\prime \prime} \mathrm{E}$ & 2 \\
\hline R. formosae & Pingtung & Pingtung & Taiwan & $22^{\circ} 40^{\prime} 16^{\prime \prime} \mathrm{N}$ & $120^{\circ} 29^{\prime} 17^{\prime \prime} \mathrm{E}$ & \\
\hline R. formosae & Kaohsiung & Kaohsiung & Taiwan & $23^{\circ} 01^{\prime} 18^{\prime \prime} \mathrm{N}$ & $120^{\circ} 39^{\prime} 25^{\prime \prime} \mathrm{E}$ & \\
\hline R. affinis & Shiyan cave & $\begin{array}{l}\text { Liping village, Jinggangshan } \\
\text { Natural Reserve }\end{array}$ & Jiangxi Province & $26^{\circ} 36^{\prime} \mathrm{N}$ & $114^{\circ} 12^{\prime} \mathrm{E}$ & 3 \\
\hline R. pearsoni & Shiyan cave & $\begin{array}{l}\text { Liping village, Jinggangshan } \\
\text { Natural Reserve }\end{array}$ & Jiangxi Province & $26^{\circ} 36^{\prime} \mathrm{N}$ & $114^{\circ} 12^{\prime} \mathrm{E}$ & \\
\hline R. macrotis & Longxu cave & Shuanghe township & Yunnan Province & $24^{\circ} 30^{\prime} \mathrm{N}$ & $102^{\circ} 20^{\prime} \mathrm{E}$ & 4 \\
\hline R. lepidus & Longxu cave & Shuanghe township & Yunnan Province & $24^{\circ} 30^{\prime} \mathrm{N}$ & $102^{\circ} 20^{\prime} \mathrm{E}$ & \\
\hline R. sinisus & Longxu cave & Shuanghe township & Yunnan Province & $24^{\circ} 30^{\prime} \mathrm{N}$ & $102^{\circ} 20^{\prime} \mathrm{E}$ & \\
\hline R. pusillus & Longxu cave & Shuanghe township & Yunnan Province & $24^{\circ} 30^{\prime} \mathrm{N}$ & $102^{\circ} 20^{\prime} \mathrm{E}$ & \\
\hline R. ferrumequinum & Longxu cave & Shuanghe township & Yunnan Province & $24^{\circ} 30^{\prime} \mathrm{N}$ & $102^{\circ} 20^{\prime} \mathrm{E}$ & \\
\hline R. rex & Longxu cave & Shuanghe township & Yunnan Province & $24^{\circ} 30^{\prime} \mathrm{N}$ & $102^{\circ} 20^{\prime} \mathrm{E}$ & \\
\hline R. rex & Qianling Park & Guiyang & Guizhou province & $26^{\circ} 36^{\prime} 58.0^{\prime \prime} \mathrm{N}$ & $106^{\circ} 41^{\prime} 26.6^{\prime \prime E}$ & 5 \\
\hline R. ferrumequinum & Dalazi Cave & Zhi'an village, Ji'an city & Jilin Province & $41^{\circ} 3^{\prime} 55.8^{\prime \prime} \mathrm{N}$ & $125^{\circ} 50^{\prime} 9.8^{\prime \prime} \mathrm{E}$ & 6 \\
\hline R. affinis & & $\begin{array}{l}\text { Jing Xi County Nature } \\
\text { Preserve }\end{array}$ & $\begin{array}{l}\text { Guangxi Autonomous } \\
\text { Region }\end{array}$ & $23 \circ 07^{\prime} 12^{\prime \prime} \mathrm{N}$ & $105 \circ 57^{\prime} 36^{\prime \prime} \mathrm{E}$ & 7 \\
\hline R. affinis & & $\begin{array}{l}\text { Shiwandashan National } \\
\text { Nature Preserve }\end{array}$ & $\begin{array}{l}\text { Guangxi Autonomous } \\
\text { Region }\end{array}$ & $21 \circ 13^{\prime} 48^{\prime \prime} N$ & $107 \circ 52^{\prime} 48^{\prime \prime} \mathrm{E}$ & \\
\hline R. pearsonii & & Dashahe Nature Preserve & Guizhou Province & $29 \circ 10^{\prime} 12^{\prime \prime} \mathrm{N}$ & $107 \circ 34^{\prime} 12^{\prime \prime} \mathrm{E}$ & \\
\hline R. rouxii & & $\begin{array}{l}\text { Shiwandashan National } \\
\text { Nature Preserve }\end{array}$ & $\begin{array}{l}\text { Guangxi Autonomous } \\
\text { Region }\end{array}$ & $21 \circ 13^{\prime} 48^{\prime \prime} \mathrm{N}$ & $107 \circ 52^{\prime} 48^{\prime \prime} \mathrm{E}$ & \\
\hline R. rouxii & Shuipu & $\begin{array}{l}\text { Maolan National Nature } \\
\text { Preserve }\end{array}$ & Guizhou Province & $25^{\circ} 29^{\prime} 05^{\prime \prime} \mathrm{N}$ & $107 \circ 52^{\prime} 54^{\prime \prime} \mathrm{E}$ & \\
\hline R. ferrumequinum & & Huizhe & Yunnan & $26: 28: 53$ & 103:27:06 & 8 \\
\hline R. ferrumequinum & & Baoxing & Sichuan & $30: 32: 57$ & $102: 42: 38$ & \\
\hline R. ferrumequinum & & Emei Shan & Sichuan & $29: 34: 74$ & 103:16:92 & \\
\hline R. ferrumequinum & & Nanton & Sichuan & $29: 48: 33$ & $102: 13: 52$ & \\
\hline
\end{tabular}


medRxiv preprint doi: https://doi.org/10.1101/2020.07.31.20166090; this version posted August 4, 2020. The copyright holder for this preprint (which was not certified by peer review) is the author/funder, who has granted medRxiv a license to display the preprint in

It is made available under a CC-BY-NC-ND 4.0 International license.

\begin{tabular}{|c|c|c|c|c|c|c|}
\hline R. ferrumequinum & & Baiya & Shaanxi & $33: 21: 32$ & 108:03:36 & \\
\hline R. ferrumequinum & & Foping & Shaanxi & $33: 34: 00$ & $107: 59: 00$ & \\
\hline R. ferrumequinum & & Shuangtai & Hubei & $32: 28: 20$ & $110: 13: 32$ & \\
\hline R. ferrumequinum & & Shuidong & Hubei & $32: 57: 56$ & $110: 25: 06$ & \\
\hline R. ferrumequinum & & Tiangou & Hubei & $32: 25: 24$ & 110:08:50 & \\
\hline R. ferrumequinum & & Shenxian & Henan & $34: 41: 49$ & $113: 22: 05$ & \\
\hline R. ferrumequinum & & Shuanglongwan & Henan & $33: 58: 28$ & $110: 56: 57$ & \\
\hline R. ferrumequinum & & Yingyang & Henan & 34:01:19 & 113:14:40 & \\
\hline R. ferrumequinum & & Zhangjiashan & Henan & $34: 28: 29$ & $110: 48: 19$ & \\
\hline R. ferrumequinum & & Jindezhen & Jiangxi & 29:19:44 & $117: 11: 48$ & \\
\hline R. ferrumequinum & & Wanfu & Jiangxi & $28: 05: 47$ & 117:01:46 & \\
\hline R. ferrumequinum & & Jinzhai & Anhui & $31: 25: 05$ & $115: 44: 44$ & \\
\hline R. ferrumequinum & & Youkuang & Anhui & $31: 10: 01$ & 115:19:59 & \\
\hline R. ferrumequinum & & Changshan & Shandong & $34: 59: 37$ & 117:58:44 & \\
\hline R. ferrumequinum & & Nanzhi & Shandong & $36: 00: 08$ & 117:00:04 & \\
\hline R. ferrumequinum & & Xiaya & Shandong & $36: 15: 00$ & 118:07:01 & \\
\hline R. ferrumequinum & & Yinan & Shandong & $35: 31: 06$ & $118: 24: 40$ & \\
\hline R. ferrumequinum & & Beijing & Beijing & $39: 54: 25$ & 116:19:55 & \\
\hline R. ferrumequinum & & Fangshan & Beijing & $39: 45: 54$ & 115:58:56 & \\
\hline R. ferrumequinum & & Jietai & Beijing & $39: 54: 35$ & $116: 14: 55$ & \\
\hline R. ferrumequinum & & Jilin Shi & Jilin & $43: 46: 49$ & $126: 29: 56$ & \\
\hline R. marshalli & Tiantang Cave & $\begin{array}{l}\text { Tiantang village, Nanning } \\
\text { city }\end{array}$ & Guangxi Province & $4183955.80 \mathrm{~N}$ & $12585099.80 \mathrm{E}$ & \\
\hline R. ferrumequinum & & Emei Shan & Sichuan & $29^{\circ} 34^{\prime} 74^{\prime \prime} \mathrm{N}$ & $103^{\circ} 16^{\prime} 92^{\prime \prime} \mathrm{E}$ & \\
\hline R. ferrumequinum & & Foping & Shaanxi & $33^{\circ} 34^{\prime} 00^{\prime \prime} \mathrm{N}$ & $107^{\circ} 59^{\prime} 00^{\prime \prime} \mathrm{E}$ & \\
\hline R. ferrumequinum & & Daguping & Shaanxi & $33^{\circ} 34^{\prime} 00^{\prime \prime} \mathrm{N}$ & $107^{\circ} 46^{\prime} 00^{\prime \prime} \mathrm{E}$ & \\
\hline R. ferrumequinum & & Beijing & Hebei & $39^{\circ} 54^{\prime} 25^{\prime \prime} \mathrm{N}$ & $116^{\circ} 19^{\prime} 55^{\prime \prime} \mathrm{E}$ & \\
\hline R. ferrumequinum & Fish cave & & Anhui Province & $30 u 209 N$ & 117u509E & \\
\hline R. sinicus & & Qingyang, Anhui & Qingyang, Anhui & N30:20:511 & E117:50:128 & \\
\hline R. sinicus & & Jingxian, Anhui & Jingxian, Anhui & N30:26:785 & E118:24:783 & \\
\hline R. sinicus & & Huashanmiku, Anhui & Huashanmiku, Anhui & N29:45:199 & E118:23:331 & \\
\hline R. sinicus & & Sanling mountain, Jiangxi & $\begin{array}{l}\text { Sanling mountain, } \\
\text { Jiangxi }\end{array}$ & $\mathrm{N} 29: 22: 112$ & E117:34:324 & \\
\hline R. sinicus & & Qingfeng cave, Jiangxi & Qingfeng cave, Jiangxi & $\mathrm{N} 29: 22: 262$ & E117:39:357 & \\
\hline R. sinicus & & Qinhui cave, Jiangxi & Qinhui cave, Jiangxi & $\mathrm{N} 29: 22: 662$ & E117:32:335 & \\
\hline R. sinicus & & Longhu mountain, Jiangxi & $\begin{array}{l}\text { Longhu mountain, } \\
\text { Jiangxi }\end{array}$ & N28:04:107 & E116:58:227 & \\
\hline R. sinicus & & Lijia country, Jiangxi & Lijia country, Jiangxi & N28:06:726 & E116:59:282 & \\
\hline R. sinicus & & Wuyishan baohuqu, Fujian & $\begin{array}{l}\text { Wuyishan baohuqu, } \\
\text { Fujian }\end{array}$ & $\mathrm{N} 27: 44: 000$ & E117:40:000 & \\
\hline R. sinicus & & Wuyishan tiliqiao, Fujian & $\begin{array}{l}\text { Wuyishan tiliqiao, } \\
\text { Fujian }\end{array}$ & $\mathrm{N} 27: 44: 543$ & E117:29:953 & \\
\hline R. sinicus & & Wuyishan Yanzijiao, Fujian & $\begin{array}{l}\text { Wuyishan Yanzijiao, } \\
\text { Fujian }\end{array}$ & $\mathrm{N} 27: 48: 511$ & E117:42:505 & \\
\hline R. sinicus & & Taihe, Jiangxi province & Taihe, Jiangxi province & $\mathrm{N} 26: 36: 151$ & E114:12:734 & \\
\hline R. sinicus & & Jinggang mountain, Jiangxi & $\begin{array}{l}\text { Jinggang mountain, } \\
\text { Jiangxi }\end{array}$ & $\mathrm{N} 26: 31: 215$ & E115:06:610 & \\
\hline R. sinicus & & Xingguo, Jiangxi & Xingguo, Jiangxi & N26:19:314 & E115:35:229 & \\
\hline R. sinicus & & Taining, Fujian & Taining, Fujian & $\mathrm{N} 26: 42: 236$ & E117:29:867 & \\
\hline R. sinicus & & Jiangle, Fujian & Jiangle, Fujian & N26:39:537 & E117:34:387 & \\
\hline R. sinicus & & Mingxi, Fujian & Mingxi, Fujian & $\mathrm{N} 26: 21: 440$ & E117:11:398 & \\
\hline R. sinicus & & Yongan, Fujian & Yongan, Fujian & $\mathrm{N} 25: 51: 500$ & E117:17:000 & \\
\hline R. sinicus & & Liancheng, Fujian & Liancheng, Fujian & $\mathrm{N} 25: 12: 404$ & E117:15:066 & \\
\hline R. sinicus & & Shanghang, Fujian & Shanghang, Fujian & $\mathrm{N} 25: 15: 020$ & E116:49:009 & \\
\hline R. sinicus & & Guilin, Guangxi & Guilin, Guangxi & $\mathrm{N} 25: 16: 278$ & E110:17:009 & \\
\hline R. sinicus & & Ruyuan, Guangdong & Ruyuan, Guangdong & $\mathrm{N} 24: 59: 086$ & E113:08:523 & \\
\hline R. sinicus & & Luofushan, Guangdong & Luofushan, Guangdong & N23:15:589 & E114:03:656 & \\
\hline R. sinicus & & Zhangjiajie, Hunan & Zhangjiajie, Hunan & $\mathrm{N} 29: 21: 410$ & E110:34:783 & \\
\hline R. sinicus & & Yongshun, Hunan & Yongshun, Hunan & $\mathrm{N} 29: 03: 720$ & E109:38:358 & \\
\hline R. sinicus & & Jishou, Hunan & Jishou, Hunan & $\mathrm{N} 28: 18: 208$ & E109:39:175 & \\
\hline R. sinicus & & Fenghuang, Hunan & Fenghuang, Hunan & N27:59:580 & E109:33:786 & \\
\hline
\end{tabular}


medRxiv preprint doi: https://doi.org/10.1101/2020.07.31.20166090; this version posted August 4, 2020. The copyright holder for this preprint (which was not certified by peer review) is the author/funder, who has granted medRxiv a license to display the preprint in perpetuity.

It is made available under a CC-BY-NC-ND 4.0 International license .

\begin{tabular}{|c|c|c|c|c|c|c|}
\hline R. sinicus & & Wuchuan, Guizhou & Wuchuan, Guizhou & $\mathrm{N} 28: 34: 237$ & E107:54:058 & \\
\hline R. sinicus & & Anlong, Guizhou & Anlong, Guizhou & $\mathrm{N} 25: 16: 577$ & E105:31:931 & \\
\hline R. sinicus & & Emeishan, Sichuan & Emeishan, Sichuan & N29:34:803 & E103:24:708 & \\
\hline R. sinicus & & Huize, Yunnan & Huize, Yunnan & N26:42:000 & E103:30:000 & \\
\hline R. sinicus & & Jiuxiang, Yunnan & Jiuxiang, Yunnan & N25:07:000 & E103:22:000 & \\
\hline R. sinicus & & Fumin, Yunnan & Fumin, Yunnan & N25:11:796 & E102:27:863 & \\
\hline R. sinicus & & Yongde, Yunnan & Yongde, Yunnan & N24:21:427 & E099:02:161 & \\
\hline R. sinicus & & Yinggeling, Hainan & Yinggeling, Hainan & N19:04:982 & E109:33:107 & \\
\hline R. sinicus & & Wuzhishan, Hainan & Wuzhishan, Hainan & N18:46:309 & E109:31:012 & \\
\hline R. sinicus & & Qiongzhong, Hainan & Qiongzhong, Hainan & $\mathrm{N} 18: 49: 589$ & E110:00:435 & \\
\hline R. sinicus & & Baoqing, Hainan & Baoqing, Hainan & $\mathrm{N} 18: 42: 237$ & E109:41:528 & \\
\hline R. sinicus & & Lingshui, Hainan & Lingshui, Hainan & $\mathrm{N} 18: 38: 586$ & E109:57:636 & \\
\hline R. sinicus & & Jianfengling, Hainan & Jianfengling, Hainan & N18:47:275 & E108:57:364 & \\
\hline R. sinicus & & Maogan, Hainan & Maogan, Hainan & $\mathrm{N} 18: 36: 306$ & E109:26:776 & \\
\hline R. affinis & Shiyan cave & $\begin{array}{l}\text { Liping village, Jinggangshan } \\
\text { Natural Reserve }\end{array}$ & Jiangxi Province & $26^{\circ} 360 \mathrm{~N}$ & $114^{\circ} 120 \mathrm{E}$ & 13 \\
\hline R. pearsoni & Shiyan cave & $\begin{array}{l}\text { Liping village, Jinggangshan } \\
\text { Natural Reserve }\end{array}$ & Jiangxi Province & $26^{\circ} 360 \mathrm{~N}$ & $114^{\circ} 120 \mathrm{E}$ & \\
\hline R. macrotis & & Yangchun & Guangdong Province & 111.94 & 22.44 & 14 \\
\hline R. macrotis & & Shaoguan & Guangdong Province & 113.56 & 24.77 & \\
\hline R. macrotis & & Nanning & Guangxi Province & 108.70 & 22.82 & \\
\hline R. macrotis & & Lengshuijiang & Hunan Province & 111.57 & 27.75 & \\
\hline R. macrotis & & Jianggangshan & Jiangxi Province & 114.20 & 26.60 & \\
\hline R. macrotis & & Jinning & Jinning & 102.33 & 24.50 & \\
\hline R. macrotis & & Yuanjiang & Yunnan Province & 102.00 & 23.59 & \\
\hline R. macrotis & & Baoshan & Yunnan Province & 99.10 & 25.08 & \\
\hline R. macrotis & & Wulong & Chongqing City & 107.95 & 29.27 & \\
\hline R. macrotis & & Yangchun & Guangdong Province & 111.94 & 22.44 & \\
\hline R. macrotis & & Xishui & Guizhou Province & 106.21 & 28.45 & \\
\hline R. macrotis & & Dazhou & Sichuan Province & 107.74 & 31.20 & \\
\hline R. macrotis & & Hanzhong & Shannxi Province & 107.03 & 32.84 & \\
\hline R. macrotis & & Xichuan & Henan Province & 111.55 & 32.87 & \\
\hline R. macrotis & & Wulong & Chongqing City & 107.95 & 29.27 & \\
\hline R. macrotis & & Lengshuijiang & Hunan Province & 111.57 & 27.75 & \\
\hline R. macrotis & & Jianggangshan & Jiangxi Province & 114.20 & 26.60 & \\
\hline R. macrotis & & Ganzhou & Jiangxi Province & 114.09 & 25.46 & \\
\hline R. macrotis & & Hanzhong & Shannxi Province & 107.03 & 32.84 & \\
\hline R. sinicus & & $\begin{array}{l}\text { 10km Mojiang, Zhenyuan, } \\
\text { and Ning'er towns, Mojiang }\end{array}$ & Yunnan Province & & & 15 \\
\hline R. affinis & & $\begin{array}{l}\text { 10km Mojiang, Zhenyuan, } \\
\text { and Ning'er towns, Mojiang }\end{array}$ & Yunnan Province & & & \\
\hline R. schnitzleri & Xiao-dong Cave & $\begin{array}{l}\text { Gengjiaying Commune, Yi- } \\
\text { liang County, Kunming City }\end{array}$ & Yunnan province & $25^{\circ} 02^{\prime} \mathrm{N}$ & $103^{\circ} 14^{\prime} \mathrm{E}$ & 16 \\
\hline R. affinis & Longkong Cave & Yanshi County & Fujian & $25^{\circ} 12^{\prime} \mathrm{N}$ & $117^{\circ} 15^{\prime} \mathrm{E}$ & 17 \\
\hline R. affinis & Zhuxi Cave & Shanghang County & Fujian & $25^{\circ} 10^{\prime} \mathrm{N}$ & $116^{\circ} 45^{\prime} \mathrm{E}$ & \\
\hline R. affinis & Jinkuang Cave & Liancheng County & Fujian & $25^{\circ} 39^{\prime} \mathrm{N}$ & $116^{\circ} 53^{\prime} \mathrm{E}$ & \\
\hline R. affinis & Qixian Cave & Sha County & Fujian & $26^{\circ} 25^{\prime} \mathrm{N}$ & $117^{\circ} 39^{\prime} \mathrm{E}$ & \\
\hline R. affinis & Guixian Cave & Mingxi County & Fujian & $26^{\circ} 24^{\prime} \mathrm{N}$ & $117^{\circ} 11^{\prime} \mathrm{E}$ & \\
\hline R. affinis & $\begin{array}{l}\text { Huanghuacong } \\
\text { Cave }\end{array}$ & $\begin{array}{l}\text { Yanzijiao Town, Wuyi } \\
\text { Mountain }\end{array}$ & Fujian & $27^{\circ} 49^{\prime} \mathrm{N}$ & $117^{\circ} 43^{\prime} \mathrm{E}$ & \\
\hline R. affinis & Shui Cave & Longmen County & Guangdong & & & \\
\hline R. affinis & Dongbian Cave & Longmen County & Guangdong & & & \\
\hline R. affinis & Penglai Cave & Yunfu Mount, Bolo County & Guangdong & & & \\
\hline R. affinis & Fangkong & $\begin{array}{l}\text { Luofu Mountain, Huizhou } \\
\text { City }\end{array}$ & Guangdong & $23^{\circ} 16^{\prime} \mathrm{N}$ & $114^{\circ} 04^{\prime} \mathrm{E}$ & \\
\hline R. affinis & Seven Star Cave & Guilin City & Guangxi & $25^{\circ} 17^{\prime} \mathrm{N}$ & $110^{\circ} 18^{\prime} \mathrm{E}$ & \\
\hline
\end{tabular}


medRxiv preprint doi: https://doi.org/10.1101/2020.07.31.20166090; this version posted August 4, 2020. The copyright holder for this preprint (which was not certified by peer review) is the author/funder, who has granted medRxiv a license to display the preprint in It is made available under a CC-BY-NC-ND 4.0 International license .

\begin{tabular}{|c|c|c|c|c|c|}
\hline R. affinis & Fenkeng Cave & Guilin City & Guangxi & $25^{\circ} 17^{\prime} \mathrm{N}$ & $110^{\circ} 21^{\prime} \mathrm{E}$ \\
\hline R. affinis & Seven Star Cave & Guilin City & Guangxi & $25^{\circ} 17^{\prime} \mathrm{N}$ & $110^{\circ} 18^{\prime} \mathrm{E}$ \\
\hline R. affinis & Bianfu Cave & Lingshui County & Hainan & $18^{\circ} 42^{\prime} \mathrm{N}$ & $109^{\circ} 53^{\prime} \mathrm{E}$ \\
\hline R. affinis & Xiashui Cave & Qiongzhong County & Hainan & $19^{\circ} 04^{\prime} \mathrm{N}$ & \\
\hline R. affinis & Qianlong Cave & Baoting County & Hainan & $18^{\circ} 34^{\prime} \mathrm{N}$ & $109^{\circ} 26^{\prime} \mathrm{E}$ \\
\hline R. affinis & Fangkong Cave & Yingge Mountain & Hainan & $19^{\circ} 04^{\prime} \mathrm{N}$ & $109^{\circ} 33^{\prime} \mathrm{E}$ \\
\hline R. affinis & Qishier Cave & $\begin{array}{l}\text { Huoshan Mountain, Haikou } \\
\text { City }\end{array}$ & Hainan & $19^{\circ} 57^{\prime} \mathrm{N}$ & $110^{\circ} 12^{\prime} \mathrm{E}$ \\
\hline R. affinis & Bianfu Cave & Xixia countryside & Henan & & \\
\hline R. affinis & $\begin{array}{l}\text { Longhushan } \\
\text { Park }\end{array}$ & Yingtan City & Jiangxi & $28^{\circ} 04^{\prime} \mathrm{N}$ & $116^{\circ} 58^{\prime} \mathrm{E}$ \\
\hline R. affinis & Jinuo Town & Xishuang banna & Yunnan & $21^{\circ} 58^{\prime} \mathrm{N}$ & $100^{\circ} 49^{\prime} \mathrm{E}$ \\
\hline R. affinis & & $\begin{array}{l}\text { Manfa Village, Xishuang } \\
\text { banna }\end{array}$ & Yunnan & & \\
\hline R. affinis & Dashu Cave & Xishuangbanna & Yunnan & & \\
\hline R. affinis & Jiuxiang Cave & Yiliang County & Yunnan & & \\
\hline R. affinis & $\begin{array}{l}\text { Shaft Cave, near } \\
\text { Bailong Cave }\end{array}$ & Mile County & Yunnan & $24^{\circ} 11^{\prime} \mathrm{N}$ & $103^{\circ} 21^{\prime} \mathrm{E}$ \\
\hline R. ferrumequinum & Bianfu Cave & SW of Beijing & Beijing & $39^{\circ} 43^{\prime} \mathrm{N}$ & $115^{\circ} 43^{\prime} \mathrm{E}$ \\
\hline R. ferrumequinum & Shidu Caves & Fangshan District & Beijing & $39^{\circ} 25^{\prime} \mathrm{N}$ & $115^{\circ} 17^{\prime} \mathrm{E}$ \\
\hline R. ferrumequinum & Bianfu Cave & SW of Beijing & Beijing & $39^{\circ} 43^{\prime} \mathrm{N}$ & $115^{\circ} 43^{\prime} \mathrm{E}$ \\
\hline R. ferrumequinum & $\begin{array}{l}\text { Bianfu Cave, } \\
\text { Beijing }\end{array}$ & Bianfu Cave, Beijing & Beijing & & \\
\hline R. ferrumequinum & $\begin{array}{l}\text { Guanyin Cave, } \\
\text { Jietai Temple }\end{array}$ & Fang shan Town & Beijing & $39^{\circ} 48^{\prime} \mathrm{N}$ & $115^{\circ} 48^{\prime} \mathrm{E}$ \\
\hline R. ferrumequinum & Seven Star Cave & Guilin City & Guangxi & $25^{\circ} 17^{\prime} \mathrm{N}$ & $110^{\circ} 18^{\prime} \mathrm{E}$ \\
\hline R. ferrumequinum & Shenxian Cave & $\begin{array}{l}\text { Huancuigu, Rongyang } \\
\text { County }\end{array}$ & Henan & $34^{\circ} 38^{\prime} \mathrm{N}$ & $113^{\circ} 15^{\prime} \mathrm{E}$ \\
\hline R. ferrumequinum & & Shennongjia & Henan & $31^{\circ} 30^{\prime} \mathrm{N}$ & $110^{\circ} 23^{\prime} \mathrm{E}$ \\
\hline R. ferrumequinum & Sihe Village & $\begin{array}{l}\text { Langhe Town, Wudang } \\
\text { Mountain }\end{array}$ & Henan & & \\
\hline R. ferrumequinum & Tong mu Village & $\begin{array}{l}\text { Muyu Town, Tanjiawan } \\
\text { County }\end{array}$ & Henan & $31^{\circ} 28^{\prime} \mathrm{N}$ & $110^{\circ} 25^{\prime} \mathrm{E}$ \\
\hline R. ferrumequinum & Wanfu Cave & Jing dezhen County & Jiangxi & $28^{\circ} 37^{\prime} \mathrm{N}$ & $116^{\circ} 28^{\prime} \mathrm{E}$ \\
\hline R. ferrumequinum & Juda Lizi Cave & Zian Village, Jian City & Jilin & $41^{\circ} 04^{\prime} \mathrm{N}$ & $125^{\circ} 50^{\prime} \mathrm{E}$ \\
\hline R. ferrumequinum & Xiaya Cave & Yiyuan County & Shandong & & \\
\hline R. ferrumequinum & & $\begin{array}{l}\text { Nantou Village, Luding } \\
\text { County }\end{array}$ & Sichuan & $29^{\circ} 48^{\prime} \mathrm{N}$ & $102^{\circ} 14^{\prime} \mathrm{E}$ \\
\hline R. ferrumequinum & Dahu Cave & Emeishan & Sichuan & $29^{\circ} 35^{\prime} \mathrm{N}$ & $103^{\circ} 17^{\prime} \mathrm{E}$ \\
\hline R. ferrumequinum & Du Cave & $\begin{array}{l}\text { Panshan Mountain, Tianjin } \\
\text { City }\end{array}$ & Tianjin & $25^{\circ} 33^{\prime} \mathrm{N}$ & $116^{\circ} 60^{\prime} \mathrm{E}$ \\
\hline R. ferrumequinum & Heshang Cave & Kunming, Fumin County & Yunnan & $25^{\circ} 12^{\prime} \mathrm{N}$ & $102^{\circ} 28^{\prime} \mathrm{E}$ \\
\hline R. ferrumequinum & Xiaogou Cave & Kunming City & Yunnan & $25^{\circ} 04^{\prime} \mathrm{N}$ & $103^{\circ} 23^{\prime} \mathrm{E}$ \\
\hline R. ferrumequinum & Xiao Cave & Yiliang County & Yunnan & & \\
\hline R. lepidus & Tianlong Cave & Dali County & Yunnan & $25^{\circ} 55^{\prime} \mathrm{N}$ & $100^{\circ} 05^{\prime} \mathrm{E}$ \\
\hline R. lepidus & Xiyou Cave & Fumin County & Yunnan & $25^{\circ} 09^{\prime} \mathrm{N}$ & $102^{\circ} 39^{\prime} \mathrm{E}$ \\
\hline R. osgoodi & Tianlong Cave & Dali County & Yunnan & $25^{\circ} 55^{\prime} \mathrm{N}$ & $100^{\circ} 05^{\prime} \mathrm{E}$ \\
\hline R. osgoodi & Xiyou Cave & Fumin County & Yunnan & $25^{\circ} 09^{\prime} \mathrm{N}$ & $102^{\circ} 39^{\prime} \mathrm{E}$ \\
\hline R. luctus & Jiuba Cave & Jiangle County & Fujian & $26^{\circ} 43^{\prime} \mathrm{N}$ & $117^{\circ} 29^{\prime} \mathrm{E}$ \\
\hline
\end{tabular}


medRxiv preprint doi: https://doi.org/10.1101/2020.07.31.20166090; this version posted August 4, 2020. The copyright holder for this preprint (which was not certified by peer review) is the author/funder, who has granted medRxiv a license to display the preprint in It is made available under a CC-BY-NC-ND 4.0 International license .

\begin{tabular}{|c|c|c|c|c|c|}
\hline R. luctus & Kangtou Cave & & Fujian & $26^{\circ} 40^{\prime} \mathrm{N}$ & $117^{\circ} 36^{\prime} \mathrm{E}$ \\
\hline R. luctus & Xianren Cave & Shishan Town, Haikou City & Hainan & $19^{\circ} 44^{\prime} \mathrm{N}$ & $109^{\circ} 37^{\prime} \mathrm{E}$ \\
\hline R. luctus & Tangle Cave & Jishou & Hunan & $28^{\circ} 18^{\prime} \mathrm{N}$ & $109^{\circ} 39^{\prime} \mathrm{E}$ \\
\hline R. macrotis & Shidu Caves & Beijing & Beijing & & \\
\hline R. macrotis & Closed Cave & Ziyuan County & Guangxi & & \\
\hline R. macrotis & Yuanfeng Cave & Qilai County & Sichuan & $30^{\circ} 29^{\prime} \mathrm{N}$ & $103^{\circ} 24^{\prime} \mathrm{E}$ \\
\hline R. siamensis & Yinhua Cave & Jiangle County & Fujian & $26^{\circ} 39^{\prime} \mathrm{N}$ & $117^{\circ} 34^{\prime} \mathrm{E}$ \\
\hline R. siamensis & Zhuxi Cave & Shanghang County & Fujian & $25^{\circ} 10^{\prime} \mathrm{N}$ & $116^{\circ} 45^{\prime} \mathrm{E}$ \\
\hline R. siamensis & Ganru Cave & Liancheng County & Fujian & & \\
\hline R. siamensis & Yinhua Cave & Jiangle County & Fujian & $26^{\circ} 39^{\prime} \mathrm{N}$ & $117^{\circ} 34^{\prime} \mathrm{E}$ \\
\hline R. siamensis & Seven Star Cave & Guilin City & Guangxi & $25^{\circ} 17^{\prime} \mathrm{N}$ & $110^{\circ} 18^{\prime} \mathrm{E}$ \\
\hline R. siamensis & Closed Cave & Ziyuan County & Guangxi & & \\
\hline R. siamensis & Wolong Cave & Xingyi County & Guizhou & $24^{\circ} 9^{\prime} \mathrm{N}$ & $104^{\circ} 53^{\prime} \mathrm{E}$ \\
\hline R. siamensis & Shanjia Cave & Anlong County & Guizhou & $25^{\circ} 19^{\prime} \mathrm{N}$ & $105^{\circ} 05^{\prime} \mathrm{E}$ \\
\hline R. siamensis & Wanfu Cave & Jing dezhen County & Jiangxi & $28^{\circ} 37^{\prime} \mathrm{N}$ & $116^{\circ} 28^{\prime} \mathrm{E}$ \\
\hline R. siamensis & & $\begin{array}{l}\text { Manfa Village, Xishuang } \\
\text { banna }\end{array}$ & Yunnan & & \\
\hline R. siamensis & Heshang Cave & Kunming, Fumin County & Yunnan & $25^{\circ} 12^{\prime} \mathrm{N}$ & $102^{\circ} 28^{\prime} \mathrm{E}$ \\
\hline R. marshalli & Baisha Cave & Guiping County & Guangxi & $23^{\circ} 14^{\prime} \mathrm{N}$ & $109^{\circ} 54^{\prime} \mathrm{E}$ \\
\hline R. marshalli & Shepo Cave & Guiping County & Guangxi & $23^{\circ} 25^{\prime} \mathrm{N}$ & $110^{\circ} 15^{\prime} \mathrm{E}$ \\
\hline R. marshalli & Bailong Cave & Mile County & Yunnan & $24^{\circ} 12^{\prime} \mathrm{N}$ & $103^{\circ} 14^{\prime} \mathrm{E}$ \\
\hline R. marshalli & Gulong Cave & Yuanjiang County & Yunnan & $23^{\circ} 35^{\prime} \mathrm{N}$ & $101^{\circ} 58^{\prime} \mathrm{E}$ \\
\hline R. pearsonii & Yulong Cave & Qingyang County & Anhui & $30^{\circ} 21^{\prime} \mathrm{N}$ & $117^{\circ} 50^{\prime} \mathrm{E}$ \\
\hline R. pearsonii & Longkong Cave & Yanshi County & Fujian & $25^{\circ} 12^{\prime} \mathrm{N}$ & $117^{\circ} 15^{\prime} \mathrm{E}$ \\
\hline R. pearsonii & Yuhua Cave & Jiangle County & Fujian & $26^{\circ} 39^{\prime} \mathrm{N}$ & $117^{\circ} 35^{\prime} \mathrm{E}$ \\
\hline R. pearsonii & Bianfu Cave & Taining City & Fujian & $26^{\circ} 42^{\prime} \mathrm{N}$ & $117^{\circ} 30^{\prime} \mathrm{E}$ \\
\hline R. pearsonii & Ganru Cave & Liancheng County & Fujian & & \\
\hline R. pearsonii & Guwang Cave & Wuyi City & Fujian & $27^{\circ} 42^{\prime} \mathrm{N}$ & $117^{\circ} 42^{\prime} \mathrm{E}$ \\
\hline R. pearsonii & $\begin{array}{l}\text { Huanghuacong } \\
\text { Cave }\end{array}$ & $\begin{array}{l}\text { Yanzijiao Town, Wuyi } \\
\text { Mountain }\end{array}$ & Fujian & $27^{\circ} 49^{\prime} \mathrm{N}$ & $117^{\circ} 43^{\prime} \mathrm{E}$ \\
\hline R. pearsonii & Shang Cave & $\begin{array}{l}\text { Dafu Village, Meihua Town, } \\
\text { Lechang County }\end{array}$ & Guangdong & $25^{\circ} 09^{\prime} \mathrm{N}$ & $113^{\circ} 04^{\prime} \mathrm{E}$ \\
\hline R. pearsonii & Seven Star Cave & Guilin City & Guangxi & $25^{\circ} 17^{\prime} \mathrm{N}$ & $110^{\circ} 18^{\prime} \mathrm{E}$ \\
\hline R. pearsonii & Closed Cave & Ziyuan County & Guangxi & & \\
\hline R. pearsonii & & $\begin{array}{l}\text { Liangjiang Town, Wuming } \\
\text { County }\end{array}$ & Guangxi & & \\
\hline R. pearsonii & Xipo Cave & Wuchuan County & Guizhou & & \\
\hline R. pearsonii & Wanfu Cave & Jing dezhen County & Jiangxi & $28^{\circ} 37^{\prime} \mathrm{N}$ & $116^{\circ} 28^{\prime} \mathrm{E}$ \\
\hline R. pearsonii & Bao Zi Cave & Tian Quan County & Sichuan & $30^{\circ} 10^{\prime} \mathrm{N}$ & $102^{\circ} 52^{\prime} \mathrm{E}$ \\
\hline R. pearsonii & Fufeng Cave & Emeishan & Sichuan & $29^{\circ} 34^{\prime} \mathrm{N}$ & $103^{\circ} 25^{\prime} \mathrm{E}$ \\
\hline R. pearsonii & Jiulao Cave & Emeishan & Sichuan & $29^{\circ} 33^{\prime} \mathrm{N}$ & $109^{\circ} 47^{\prime} \mathrm{E}$ \\
\hline R. pearsonii & Mingjiu Cave & Mongzi County & Yunnan & & \\
\hline R. pearsonii & Pingbian Village & Mongzi County & Yunnan & & \\
\hline R. pearsonii & $\begin{array}{l}\text { Wangzhangya } \\
\text { Cave }\end{array}$ & Dawei County & Yunnan & & \\
\hline
\end{tabular}


medRxiv preprint doi: https://doi.org/10.1101/2020.07.31.20166090; this version posted August 4, 2020. The copyright holder for this preprint (which was not certified by peer review) is the author/funder, who has granted medRxiv a license to display the preprint in It is made available under a CC-BY-NC-ND 4.0 International license .

\begin{tabular}{|c|c|c|c|c|c|}
\hline R. pearsonii & Bianfu Cave & Mengla County & Yunnan & $21^{\circ} 53^{\prime} \mathrm{N}$ & $101^{\circ} 18^{\prime} \mathrm{E}$ \\
\hline R. yunanensis & Yulong Cave & Qingyang County & Anhui & $30^{\circ} 21^{\prime} \mathrm{N}$ & $117^{\circ} 50^{\prime} \mathrm{E}$ \\
\hline R. yunanensis & Longkong Cave & Yanshi County & Fujian & $25^{\circ} 12^{\prime} \mathrm{N}$ & $117^{\circ} 15^{\prime} \mathrm{E}$ \\
\hline R. yunanensis & Yuhua Cave & Jiangle County & Fujian & $26^{\circ} 39^{\prime} \mathrm{N}$ & $117^{\circ} 35^{\prime} \mathrm{E}$ \\
\hline R. yunanensis & Bianfu Cave & Taining City & Fujian & $26^{\circ} 42^{\prime} \mathrm{N}$ & $117^{\circ} 30^{\prime} \mathrm{E}$ \\
\hline R. yunanensis & Ganru Cave & Liancheng County & Fujian & & \\
\hline R. yunanensis & Guwang Cave & Wuyi City & Fujian & $27^{\circ} 42^{\prime} \mathrm{N}$ & $117^{\circ} 42^{\prime} \mathrm{E}$ \\
\hline R. yunanensis & $\begin{array}{l}\text { Huanghuacong } \\
\text { Cave }\end{array}$ & $\begin{array}{l}\text { Yanzijiao Town, Wuyi } \\
\text { Mountain }\end{array}$ & Fujian & $27^{\circ} 49^{\prime} \mathrm{N}$ & $117^{\circ} 43^{\prime} \mathrm{E}$ \\
\hline R. yunanensis & Shang Cave & $\begin{array}{l}\text { Dafu Village, Meihua Town, } \\
\text { Lechang County }\end{array}$ & Guangdong & $25^{\circ} 09^{\prime} \mathrm{N}$ & $113^{\circ} 04^{\prime} \mathrm{E}$ \\
\hline R. yunanensis & Seven Star Cave & Guilin City & Guangxi & $25^{\circ} 17^{\prime} \mathrm{N}$ & $110^{\circ} 18^{\prime} \mathrm{E}$ \\
\hline R. yunanensis & Closed Cave & Ziyuan County & Guangxi & & \\
\hline R. yunanensis & & $\begin{array}{l}\text { Liangjiang Town, Wuming } \\
\text { County }\end{array}$ & Guangxi & & \\
\hline R. yunanensis & Xipo Cave & Wuchuan County & Guizhou & & \\
\hline R. yunanensis & Wanfu Cave & Jing dezhen County & Jiangxi & $28^{\circ} 37^{\prime} \mathrm{N}$ & $116^{\circ} 28^{\prime} \mathrm{E}$ \\
\hline R. yunanensis & Bao Zi Cave & Tian Quan County & Sichuan & $30^{\circ} 10^{\prime} \mathrm{N}$ & $102^{\circ} 52^{\prime} \mathrm{E}$ \\
\hline R. yunanensis & Fufeng Cave & Emeishan & Sichuan & $29^{\circ} 34^{\prime} \mathrm{N}$ & $103^{\circ} 25^{\prime} \mathrm{E}$ \\
\hline R. yunanensis & Jiulao Cave & Emeishan & Sichuan & $29^{\circ} 33^{\prime} \mathrm{N}$ & $109^{\circ} 47^{\prime} \mathrm{E}$ \\
\hline R. yunanensis & Mingjiu Cave & Mongzi County & Yunnan & & \\
\hline R. yunanensis & Pingbian Village & Mongzi County & Yunnan & & \\
\hline R. yunanensis & $\begin{array}{l}\text { Wangzhangya } \\
\text { Cave }\end{array}$ & Dawei County & Yunnan & & \\
\hline R. yunanensis & Bianfu Cave & Mengla County & Yunnan & $21^{\circ} 53^{\prime} \mathrm{N}$ & $101^{\circ} 18^{\prime} \mathrm{E}$ \\
\hline R. rex & Dongmen Cave & Fushui County & Guangxi & & \\
\hline R. rex & $\begin{array}{l}\text { White Dragon } \\
\text { Cave }\end{array}$ & Guilin City & Guangxi & & \\
\hline R. rex & Fenkeng Cave & Guilin City & Guangxi & $25^{\circ} 17^{\prime} \mathrm{N}$ & $110^{\circ} 21^{\prime} \mathrm{E}$ \\
\hline R. rex & Fenkeng Cave & Guilin City & Guangxi & $25^{\circ} 17^{\prime} \mathrm{N}$ & $110^{\circ} 21^{\prime} \mathrm{E}$ \\
\hline R. rex & Xipo Cave & Wuchuan County & Guizhou & & \\
\hline R. rex & Qingxuan Cave & & Yunnan & $26^{\circ} 40^{\prime} \mathrm{N}$ & $100^{\circ} 12^{\prime} \mathrm{E}$ \\
\hline R. rex & Tianlong Cave & Dali County & Yunnan & $25^{\circ} 55^{\prime} \mathrm{N}$ & $100^{\circ} 05^{\prime} \mathrm{E}$ \\
\hline R. rex & Bianfu Cave & Yiliang County & Yunnan & $25^{\circ} 04^{\prime} \mathrm{N}$ & $103^{\circ} 23^{\prime} \mathrm{E}$ \\
\hline R. rex & Xiyou Cave & Fumin County & Yunnan & $25^{\circ} 09^{\prime} \mathrm{N}$ & $102^{\circ} 39^{\prime} \mathrm{E}$ \\
\hline R. rex & Xiaogou Cave & Yiliang County & Yunnan & & \\
\hline R. paradoxolophus & Dongmen Cave & Fushui County & Guangxi & & \\
\hline R. paradoxolophus & $\begin{array}{l}\text { White Dragon } \\
\text { Cave }\end{array}$ & Guilin City & Guangxi & & \\
\hline R. paradoxolophus & Fenkeng Cave & Guilin City & Guangxi & $25^{\circ} 17^{\prime} \mathrm{N}$ & $110^{\circ} 21^{\prime} \mathrm{E}$ \\
\hline R. paradoxolophus & Fenkeng Cave & Guilin City & Guangxi & $25^{\circ} 17^{\prime} \mathrm{N}$ & $110^{\circ} 21^{\prime} \mathrm{E}$ \\
\hline R. paradoxolophus & Xipo Cave & Wuchuan County & Guizhou & & \\
\hline R. paradoxolophus & Qingxuan Cave & & Yunnan & $26^{\circ} 40^{\prime} \mathrm{N}$ & $100^{\circ} 12^{\prime} \mathrm{E}$ \\
\hline R. paradoxolophus & Tianlong Cave & Dali County & Yunnan & $25^{\circ} 55^{\prime} \mathrm{N}$ & $100^{\circ} 05^{\prime} \mathrm{E}$ \\
\hline R. paradoxolophus & Bianfu Cave & Yiliang County & Yunnan & $25^{\circ} 04^{\prime} \mathrm{N}$ & $103^{\circ} 23^{\prime} \mathrm{E}$ \\
\hline R. paradoxolophus & Xiyou Cave & Fumin County & Yunnan & $25^{\circ} 09^{\prime} \mathrm{N}$ & $102^{\circ} 39^{\prime} \mathrm{E}$ \\
\hline
\end{tabular}


medRxiv preprint doi: https://doi.org/10.1101/2020.07.31.20166090; this version posted August 4, 2020. The copyright holder for this preprint (which was not certified by peer review) is the author/funder, who has granted medRxiv a license to display the preprint in It is made available under a CC-BY-NC-ND 4.0 International license .

\begin{tabular}{|c|c|c|c|c|c|}
\hline R. paradoxolophus & Xiaogou Cave & Yiliang County & Yunnan & & \\
\hline R. sinicus & Yinhua Cave & Jiangle County & Fujian & $26^{\circ} 39^{\prime} \mathrm{N}$ & $117^{\circ} 34^{\prime} \mathrm{E}$ \\
\hline R. sinicus & Bianfu Cave & Taining City & Fujian & $26^{\circ} 42^{\prime} \mathrm{N}$ & $117^{\circ} 30^{\prime} \mathrm{E}$ \\
\hline$R$. sinicus & Longkong Cave & Yanshi County & Fujian & $25^{\circ} 12^{\prime} \mathrm{N}$ & $117^{\circ} 15^{\prime} \mathrm{E}$ \\
\hline R. sinicus & Mine & $\begin{array}{l}\text { Buyun Town, Shanghang } \\
\text { County }\end{array}$ & Fujian & $25^{\circ} 15^{\prime} \mathrm{N}$ & $116^{\circ} 50^{\prime} \mathrm{E}$ \\
\hline R. sinicus & Jinkuang Cave & Liancheng County & Fujian & $25^{\circ} 39^{\prime} \mathrm{N}$ & $116^{\circ} 53^{\prime} \mathrm{E}$ \\
\hline R. sinicus & Liuhuang Cave & Liancheng County & Fujian & $25^{\circ} 52^{\prime} \mathrm{N}$ & $117^{\circ} 17^{\prime} \mathrm{E}$ \\
\hline R. sinicus & Yuxi Cave & Mingxi County & Fujian & $26^{\circ} 21^{\prime} \mathrm{N}$ & $117^{\circ} 11^{\prime} \mathrm{E}$ \\
\hline R. sinicus & Yinhua Cave & Jiangle County & Fujian & $26^{\circ} 39^{\prime} \mathrm{N}$ & $117^{\circ} 34^{\prime} \mathrm{E}$ \\
\hline R. sinicus & Xing Cave & $\begin{array}{l}\text { Qiliqiao Bridge, Wuyi } \\
\text { Mountain }\end{array}$ & Fujian & $27^{\circ} 45^{\prime} \mathrm{N}$ & $117^{\circ} 30^{\prime} \mathrm{E}$ \\
\hline$R$. sinicus & $\begin{array}{l}\text { Mine, Chang } \\
\text { ganzhou Road }\end{array}$ & Xincun Town, Wuyi City & Fujian & $27^{\circ} 37^{\prime} \mathrm{N}$ & $117^{\circ} 50^{\prime} \mathrm{E}$ \\
\hline$R$. sinicus & $\begin{array}{l}\text { Huanghuacong } \\
\text { Cave }\end{array}$ & $\begin{array}{l}\text { Yanzijiao Town, Wuyi } \\
\text { Mountain }\end{array}$ & Fujian & $27^{\circ} 49^{\prime} \mathrm{N}$ & $117^{\circ} 43^{\prime} \mathrm{E}$ \\
\hline$R$. sinicus & Penglai Cave & Yunfu Mount, Bolo County & Guangdong & & \\
\hline$R$. sinicus & Fangkong & $\begin{array}{l}\text { Luofu Mountain, Huizhou } \\
\text { City }\end{array}$ & Guangdong & $23^{\circ} 16^{\prime} \mathrm{N}$ & $114^{\circ} 04^{\prime} \mathrm{E}$ \\
\hline$R$. sinicus & Ni Cave & Xinyi County & Guangdong & $22^{\circ} 20^{\prime} \mathrm{N}$ & $110^{\circ} 57^{\prime} \mathrm{E}$ \\
\hline R. sinicus & Shuili Cave & Xinyi County & Guangdong & $22^{\circ} 20^{\prime} \mathrm{N}$ & $110^{\circ} 58^{\prime} \mathrm{E}$ \\
\hline R. sinicus & Kiln & Xinyi County & Guangdong & $22^{\circ} 15^{\prime} \mathrm{N}$ & $110^{\circ} 60^{\prime} \mathrm{E}$ \\
\hline$R$. sinicus & Closed Cave & Ziyuan County & Guangxi & & \\
\hline$R$. sinicus & Fenkeng Cave & Guilin City & Guangxi & $25^{\circ} 17^{\prime} \mathrm{N}$ & $110^{\circ} 21^{\prime} \mathrm{E}$ \\
\hline$R$. sinicus & Fenkeng Cave & Guilin City & Guangxi & $25^{\circ} 17^{\prime} \mathrm{N}$ & $110^{\circ} 21^{\prime} \mathrm{E}$ \\
\hline R. sinicus & Shui Cave & Guilin City & Guangxi & & \\
\hline$R$. sinicus & Jiang Cave & Jiangkou County & Guizhou & $23^{\circ} 25^{\prime} \mathrm{N}$ & $110^{\circ} 15^{\prime} \mathrm{E}$ \\
\hline R. sinicus & Xiashui Cave & Qiongzhong County & Hainan & $19^{\circ} 04^{\prime} \mathrm{N}$ & \\
\hline$R$. sinicus & Hela Village & $\begin{array}{l}\text { Hongmao Town, } \\
\text { Qiongzhong County }\end{array}$ & Hainan & & \\
\hline R. sinicus & Diyi Village & Five Finger Mountain & Hainan & & \\
\hline R. sinicus & Fangkong Cave & Jianfeng Mountain & Hainan & $18^{\circ} 54^{\prime} \mathrm{N}$ & $109^{\circ} 42^{\prime} \mathrm{E}$ \\
\hline$R$. sinicus & Cave & Yichang City & Hubei & & \\
\hline$R$. sinicus & $\begin{array}{l}\text { Longhushan } \\
\text { Park }\end{array}$ & Yingtan City & Jiangxi & $28^{\circ} 04^{\prime} \mathrm{N}$ & $116^{\circ} 58^{\prime} \mathrm{E}$ \\
\hline$R$. sinicus & Wanfu Cave & Jing dezhen County & Jiangxi & $28^{\circ} 37^{\prime} \mathrm{N}$ & $116^{\circ} 28^{\prime} \mathrm{E}$ \\
\hline$R$. sinicus & Fufeng Cave & Emeishan & Sichuan & $29^{\circ} 34^{\prime} \mathrm{N}$ & $103^{\circ} 25^{\prime} \mathrm{E}$ \\
\hline$R$. sinicus & Jiulao Cave & Emeishan & Sichuan & $29^{\circ} 33^{\prime} \mathrm{N}$ & $109^{\circ} 47^{\prime} \mathrm{E}$ \\
\hline$R$. sinicus & Bailong Cave & Mile County & Yunnan & $24^{\circ} 12^{\prime} \mathrm{N}$ & $103^{\circ} 14^{\prime} \mathrm{E}$ \\
\hline R. pusillus & Shidu Caves & Fangshan District & Beijing & $39^{\circ} 25^{\prime} \mathrm{N}$ & $115^{\circ} 17^{\prime} \mathrm{E}$ \\
\hline R. pusillus & Sanqing Cave & $\begin{array}{l}\text { Wanglaopu, Fangshan } \\
\text { District }\end{array}$ & Beijing & $39^{\circ} 45^{\prime} \mathrm{N}$ & $115^{\circ} 45^{\prime} \mathrm{E}$ \\
\hline R. pusillus & Longkong Cave & Yanshi County & Fujian & $25^{\circ} 12^{\prime} \mathrm{N}$ & $117^{\circ} 15^{\prime} \mathrm{E}$ \\
\hline R. pusillus & Yinhua Cave & Jiangle County & Fujian & $26^{\circ} 39^{\prime} \mathrm{N}$ & $117^{\circ} 34^{\prime} \mathrm{E}$ \\
\hline R. pusillus & Jinkuang Cave & Liancheng County & Fujian & $25^{\circ} 39^{\prime} \mathrm{N}$ & $116^{\circ} 53^{\prime} \mathrm{E}$ \\
\hline R.pusillus & Ganru Cave & Liancheng County & Fujian & & \\
\hline
\end{tabular}


medRxiv preprint doi: https://doi.org/10.1101/2020.07.31.20166090; this version posted August 4, 2020. The copyright holder for this preprint (which was not certified by peer review) is the author/funder, who has granted medRxiv a license to display the preprint in It is made available under a CC-BY-NC-ND 4.0 International license .

\begin{tabular}{|c|c|c|c|c|c|}
\hline R. pusillus & $\begin{array}{l}\text { Niutongguan } \\
\text { Cave }\end{array}$ & Liancheng County & Fujian & $25^{\circ} 33^{\prime} \mathrm{N}$ & $116^{\circ} 59^{\prime} \mathrm{E}$ \\
\hline R. pusillus & Chuqi Cave & Liancheng County & Fujian & $25^{\circ} 33^{\prime} \mathrm{N}$ & $116^{\circ} 60^{\prime} \mathrm{E}$ \\
\hline R. pusillus & Liuhuang Cave & Liancheng County & Fujian & $25^{\circ} 52^{\prime} \mathrm{N}$ & $117^{\circ} 17^{\prime} \mathrm{E}$ \\
\hline R. pusillus & Qixian Cave & Sha County & Fujian & $26^{\circ} 25^{\prime} \mathrm{N}$ & $117^{\circ} 39^{\prime} \mathrm{E}$ \\
\hline R. pusillus & Yinhua Cave & Jiangle County & Fujian & $26^{\circ} 39^{\prime} \mathrm{N}$ & $117^{\circ} 34^{\prime} \mathrm{E}$ \\
\hline R. pusillus & Jiuba Cave & Jiangle County & Fujian & $26^{\circ} 43^{\prime} \mathrm{N}$ & $117^{\circ} 29^{\prime} \mathrm{E}$ \\
\hline R. pusillus & $\begin{array}{l}\text { Cave of the } \\
\text { Buddha }\end{array}$ & $\begin{array}{l}\text { Dingushan Forest Eco } \\
\text { system, }\end{array}$ & Guangdong & & \\
\hline R. pusillus & Butterfly Cave & Yunfu Mount & Guangdong & & \\
\hline R. pusillus & Fangkong & $\begin{array}{l}\text { Luofu Mountain, Huizhou } \\
\text { City }\end{array}$ & Guangdong & $23^{\circ} 16^{\prime} \mathrm{N}$ & $114^{\circ} 04^{\prime} \mathrm{E}$ \\
\hline R. pusillus & Dianxian Cave & Xinyi County & Guangdong & $22^{\circ} 20^{\prime} \mathrm{N}$ & $110^{\circ} 57^{\prime} \mathrm{E}$ \\
\hline R. pusillus & Shuili Cave & Xinyi County & Guangdong & $22^{\circ} 20^{\prime} \mathrm{N}$ & $110^{\circ} 58^{\prime} \mathrm{E}$ \\
\hline R. pusillus & Rock crevice & Xinyi County & Guangdong & $22^{\circ} 15^{\prime} \mathrm{N}$ & $111^{\circ} 00^{\prime} \mathrm{E}$ \\
\hline R. pusillus & Tongtian Cave & Xinyi County & Guangdong & $22^{\circ} 23^{\prime} \mathrm{N}$ & $110^{\circ} 72^{\prime} \mathrm{E}$ \\
\hline R. pusillus & Chang Cave & Xinyi County & Guangdong & $22^{\circ} 17^{\prime} \mathrm{N}$ & $111^{\circ} 02^{\prime} \mathrm{E}$ \\
\hline R. pusillus & Jishui Cave & Guilin City & Guangxi & $25^{\circ} 17^{\prime} \mathrm{N}$ & $110^{\circ} 21^{\prime} \mathrm{E}$ \\
\hline R. pusillus & $\begin{array}{l}\text { White Dragon } \\
\text { Cave }\end{array}$ & Guilin City & Guangxi & & \\
\hline R. pusillus & Seven Star Cave & Guilin City & Guangxi & $25^{\circ} 17^{\prime} \mathrm{N}$ & $110^{\circ} 18^{\prime} \mathrm{E}$ \\
\hline R. pusillus & Closed Cave & Ziyuan County & Guangxi & & \\
\hline R. pusillus & & $\begin{array}{l}\text { Liangjiang Town, Wuming } \\
\text { County }\end{array}$ & Guangxi & & \\
\hline R. pusillus & Shui Cave & Guilin City & Guangxi & & \\
\hline R. pusillus & Shui Cave & Guilin City & Guangxi & & \\
\hline R. pusillus & Jishui Cave & Guilin City & Guangxi & $25^{\circ} 17^{\prime} \mathrm{N}$ & $110^{\circ} 21^{\prime} \mathrm{E}$ \\
\hline R. pusillus & Seven Star Cave & Guilin City & Guangxi & $25^{\circ} 17^{\prime} \mathrm{N}$ & $110^{\circ} 18^{\prime} \mathrm{E}$ \\
\hline R. pusillus & Wulong Cave & Mashan County & Guangxi & & \\
\hline R. pusillus & Shanjia Cave & Anlong County & Guizhou & $25^{\circ} 19^{\prime} \mathrm{N}$ & $105^{\circ} 05^{\prime} \mathrm{E}$ \\
\hline R. pusillus & & Leigong Mountain & Guizhou & & \\
\hline R. pusillus & Fangkong Cave & Jianfeng Mountain & Hainan & $18^{\circ} 54^{\prime} \mathrm{N}$ & $109^{\circ} 42^{\prime} \mathrm{E}$ \\
\hline R. pusillus & & Yichang City & Hubei & $31^{\circ} 09^{\prime} \mathrm{N}$ & $111^{\circ} 08^{\prime} \mathrm{E}$ \\
\hline R. pusillus & Shenlong Cave & Yichang & Hubei & $31^{\circ} 21^{\prime} \mathrm{N}$ & $110^{\circ} 30^{\prime} \mathrm{E}$ \\
\hline R. pusillus & Yeren Cave & Shennon gjia & Hubei & $31^{\circ} 55^{\prime} \mathrm{N}$ & $110^{\circ} 44^{\prime} \mathrm{E}$ \\
\hline R. pusillus & Sihe Village & $\begin{array}{l}\text { Langhe Town, Wudang } \\
\text { Mountain }\end{array}$ & Henan & & \\
\hline R. pusillus & Xianren Cave & Wuhan City & Hubei & $25^{\circ} 53^{\prime} \mathrm{N}$ & $114^{\circ} 30^{\prime} \mathrm{E}$ \\
\hline R. pusillus & $\begin{array}{l}\text { Longhushan } \\
\text { Park }\end{array}$ & Yingtan City & Jiangxi & $28^{\circ} 04^{\prime} \mathrm{N}$ & $116^{\circ} 58^{\prime} \mathrm{E}$ \\
\hline R. pusillus & Wanfu Cave & Jing dezhen County & Jiangxi & $28^{\circ} 37^{\prime} \mathrm{N}$ & $116^{\circ} 28^{\prime} \mathrm{E}$ \\
\hline R. pusillus & Xiaya Cave & Yiyuan County & Shandong & & \\
\hline R. pusillus & Mine & $\begin{array}{l}\text { Buyun Town, Shanghang } \\
\text { County }\end{array}$ & Shandong & $25^{\circ} 15^{\prime} \mathrm{N}$ & $116^{\circ} 50^{\prime} \mathrm{E}$ \\
\hline R. pusillus & & Yilai Mountain & Sichuan & $30^{\circ} 29^{\prime} \mathrm{N}$ & $103^{\circ} 24^{\prime} \mathrm{E}$ \\
\hline R. pusillus & & Luding County & Sichuan & $29^{\circ} 48^{\prime} \mathrm{N}$ & $102^{\circ} 14^{\prime} \mathrm{E}$ \\
\hline R. pusillus & Qilai County & Qilai County & Sichuan & & \\
\hline
\end{tabular}


medRxiv preprint doi: https://doi.org/10.1101/2020.07.31.20166090; this version posted August 4, 2020. The copyright holder for this preprint (which was not certified by peer review) is the author/funder, who has granted medRxiv a license to display the preprint in

It is made available under a CC-BY-NC-ND 4.0 International license .

\begin{tabular}{|c|c|c|c|c|c|}
\hline R. pusillus & Ahei Cave & Xishuangbanna & Yunnan & & \\
\hline R. pusillus & Heshang Cave & Kunming, Fumin County & Yunnan & $25^{\circ} 12^{\prime} \mathrm{N}$ & $102^{\circ} 28^{\prime} \mathrm{E}$ \\
\hline R. pusillus & $\begin{array}{l}\text { Xiangshui } \\
\text { Village }\end{array}$ & Fumin County & Yunnan & & \\
\hline R. pusillus & $\begin{array}{l}\text { Wangzhangya } \\
\text { Cave }\end{array}$ & Dawei County & Yunnan & & \\
\hline R. pusillus & Fengjing Cave & Jiuxiang County & Yunnan & & \\
\hline R. pusillus & Jiuxiang Cave & Yiliang County & Yunnan & & \\
\hline R. pusillus & Bailong Cave & Mile County & Yunnan & $24^{\circ} 12^{\prime} \mathrm{N}$ & $103^{\circ} 14^{\prime} \mathrm{E}$ \\
\hline R. pusillus & $\begin{array}{l}\text { Shaft Cave, near } \\
\text { Bailong Cave }\end{array}$ & Mile County & Yunnan & $24^{\circ} 11^{\prime} \mathrm{N}$ & $103^{\circ} 21^{\prime} \mathrm{E}$ \\
\hline R. stheno & Ahei Cave & Xishuang banna & Yunnan & & \\
\hline R. stheno & Jinuo Cave & Xishuang banna & Yunnan & $21^{\circ} 58^{\prime} \mathrm{N}$ & $100^{\circ} 49^{\prime} \mathrm{E}$ \\
\hline
\end{tabular}

1 Sun, K.-P. et al. A new cryptic species of rhinolophus macrotis (Chiroptera : Rhinolophidae) from Jiangxi Province, China. Acta Chiropterologica 10, 1-10, doi:10.3161/150811008×331045 (2008).

2 Volleth, M. et al. Comparative chromosomal studies in Rhinolophus formosae and R-luctus from China and Vietnam: elevation of R-I. lanosus to species rank. Acta Chiropterologica 19, 41-50, doi:10.3161/15081109acc2017.19.1.003 (2017).

3 Jiang, T., Lu, G., Sun, K., Luo, J. \& Feng, J. Coexistence of Rhinolophus affinis and Rhinolophus pearsoni revisited. Acta Theriologica 58, 47-53, doi:10.1007/s13364-012-0093-x (2013).

4 Shi, L.-m., Feng, J., Liu, Y., Ye, G.-x. \& Zhu, X. Is food resource partitioning responsible for deviation of echolocation call frequencies from allometry in Rhinolophus macrotis? Acta Theriologica 54, 371-382, doi:10.4098/j.at.0001-7051.099.2008 (2009).

5 He, G. Z. et al. Entamoeba histolytica Infections in a King Horseshoe Bat (Rhinolophus rex): A First Case Report. Asian Journal of Animal and Veterinary Advances 6, 1026-1030, doi:10.3923/ajava.2011.1026.1030 (2011).

$6 \quad$ Liu, Y., Feng, J., Jiang, Y. L., Wu, L. \& Sun, K. P. Vocalization development of greater horseshoe bat, Rhinolophus ferrumequinum (Rhinolophidae, Chiroptera). Folia Zoologica 56, 126-136 (2007).

7 Bush, S. E. \& Robbins, R. G. New host and locality records for Ixodes simplex Neumann and Ixodes vespertilionis Koch (Acari: Ixodidae) from bats (Chiroptera: Hipposideridae, Rhinolophidae and Vespertilionidae) in southern China. International Journal of Acarology 38, 1-5, doi:10.1080/01647954.2011.569509 (2012).

8 Flanders, J., Wei, L., Rossiter, S. J. \& Zhang, S. Identifying the effects of the Pleistocene on the greater horseshoe bat, Rhinolophus ferrumequinum, in East Asia using ecological niche modelling and phylogenetic analyses. Journal of Biogeography 38, 439-452, doi:10.1111/j.1365-2699.2010.02411.x (2011).

9 Liu, Y., Jiang, T., Berquist, S. \& Feng, J. Vocal characters and wing morphology of Rhinolophus marshalli from Tiantang Cave, Guangxi Province, China. Mammalia 73, 373-376, doi:10.1515/mamm.2009.053 (2009).

10 Rossiter, S. J., Benda, P., Dietz, C., Zhang, S. \& Jones, G. Rangewide phylogeography in the greater horseshoe bat inferred from microsatellites: implications for population history, taxonomy and conservation. Molecular Ecology 16, 4699-4714, doi:10.1111/j.1365-294X.2007.03546.x (2007).

11 Lei, M., Dong, D., Mu, S., Pan, Y.-H. \& Zhang, S. Comparison of Brain Transcriptome of the Greater Horseshoe Bats (Rhinolophus ferrumequinum) in Active and Torpid Episodes. Plos One 9, doi:10.1371/journal.pone.0107746 (2014).

12 Mao, X., He, G., Zhang, J., Rossiter, S. J. \& Zhang, S. Lineage Divergence and Historical Gene Flow in the Chinese Horseshoe Bat (Rhinolophus sinicus). Plos One 8, doi:10.1371/journal.pone.0056786 (2013).

13 Jiang, T., Feng, J., Sun, K. \& Wang, J. Coexistence of two sympatric and morphologically similar bat species Rhinolophus affinis and Rhinolophus pearsoni. Progress in Natural Science-Materials International 18, 523532, doi:10.1016/j.pnsc.2007.12.005 (2008).

14 Sun, K. et al. The complex evolutionary history of big-eared horseshoe bats (Rhinolophus macrotis complex): insights from genetic, morphological and acoustic data. Scientific Reports 6, doi:10.1038/srep35417 (2016).

15 Ge, X.-Y. et al. Coexistence of multiple coronaviruses in several bat colonies in an abandoned mineshaft. Virologica Sinica 31, 31-40, doi:10.1007/s12250-016-3713-9 (2016).

16 Wu, Y. \& Vu Dinh, T. A New Species of Rhinolophus (Chiroptera: Rhinolophidae) from China. Zoological Science 28, 235-241, doi:10.2108/zsj.28.235 (2011).

17 Zhang, L. et al. Recent surveys of bats (Mammalia: Chiroptera) from China. I. Rhinolophidae and Hipposideridae. Acta Chiropterologica 11, 71-88, doi:10.3161/150811009x465703 (2009). 
medRxiv preprint doi: https://doi.org/10.1101/2020.07.31.20166090; this version posted August 4, 2020. The copyright holder for this preprint (which was not certified by peer review) is the author/funder, who has granted medRxiv a license to display the preprint in It is made available under a CC-BY-NC-ND 4.0 International license

559 Table S2. Mean land cover and land use attributes (and $20 \%$ and $80 \%$ percentiles) in the 560 surroundings (<30km distance) of bat location and randomly selected points (see methods) within 561 and outside China.

\begin{tabular}{|c|c|c|c|c|c|c|c|c|c|}
\hline & \multicolumn{3}{|c|}{ Bat Locations } & \multicolumn{3}{|c|}{ Random in China } & \multicolumn{3}{|c|}{ Random out China } \\
\hline & Mean & $20 \%$ & $80 \%$ & Mean & $20 \%$ & $80 \%$ & Mean & $20 \%$ & $80 \%$ \\
\hline \multicolumn{10}{|l|}{ Livestock Density (heads/(km²) } \\
\hline - Chickens & 781 & 183 & 931 & 703 & 160 & 841 & 317 & 15 & 317 \\
\hline - Ducks & 256 & 18 & 294 & 202 & 10 & 264 & 26 & 0 & 16 \\
\hline - Pigs & 99 & 42 & 163 & 107 & 43 & 148 & 18 & 0 & 21 \\
\hline - $\quad$ Goats & 32 & 0 & 47 & 30 & 4 & 42 & 8 & 0 & 9 \\
\hline - Sheep & 5 & 0 & 0 & 3 & 0 & 1 & 14 & 0 & 21 \\
\hline - Cattle & 22 & 5 & 31 & 22 & 6 & 32 & 16 & 2 & 21 \\
\hline Forest Cover (\%) & 45 & 19 & 71 & 35 & 13 & 55 & 52 & 20 & 80 \\
\hline Forest Fragmentation () & 73 & 54 & 96 & 83 & 68 & 98 & 61 & 35 & 90 \\
\hline Cropland () & 20 & 5 & 36 & 24 & 6 & 38 & 30 & 3 & 59 \\
\hline People (cap/km²) & 282 & 63 & 318 & 199 & 59 & 270 & 107 & 12 & 114 \\
\hline Villages/Human Settlements () & 11 & 3 & 18 & 10 & 2 & 16 & 4 & 0 & 5 \\
\hline
\end{tabular}


medRxiv preprint doi: https://doi.org/10.1101/2020.07.31.20166090; this version posted August 4, 2020. The copyright holder for this preprint (which was not certified by peer review) is the author/funder, who has granted medRxiv a license to display the preprint in It is made available under a CC-BY-NC-ND 4.0 International license .

Table S3. Report for the spatially constrained multivariate grouping analysis, showing group numerosity, descriptive statistics and boxplots, in comparison with the global benchmark. The share is calculated as the ratio of the variable range (min-max) which is occupied by the same variable in a group. Q1 and Q3 are respectively the first and third quartile, and sd is the standard deviation. The R2 parameter measures the indicator's performance in grouping the sample, as relative reduction of the intra-group variability w.r.t. the global sample variability. Variables include fragmentation (frg), livestock (Ist), and settlements (stl). Boxplots are an intuitive tool to visually recap statistical distributions. The meaning of the elements of boxplots is described in the figure below. In the report, the first and third quartile are expressed respectively as Q1 and Q3. The high number of outliers for livestock and settlements is typical for high-skewness distributions. The boxplots show well how the indicator distributions shift and narrow from the global dataset to single groups. Although in some the standard deviation of one or more indicators is higher than the global benchmark, each group can be considered as homogeneous for at least

577 one parameter.

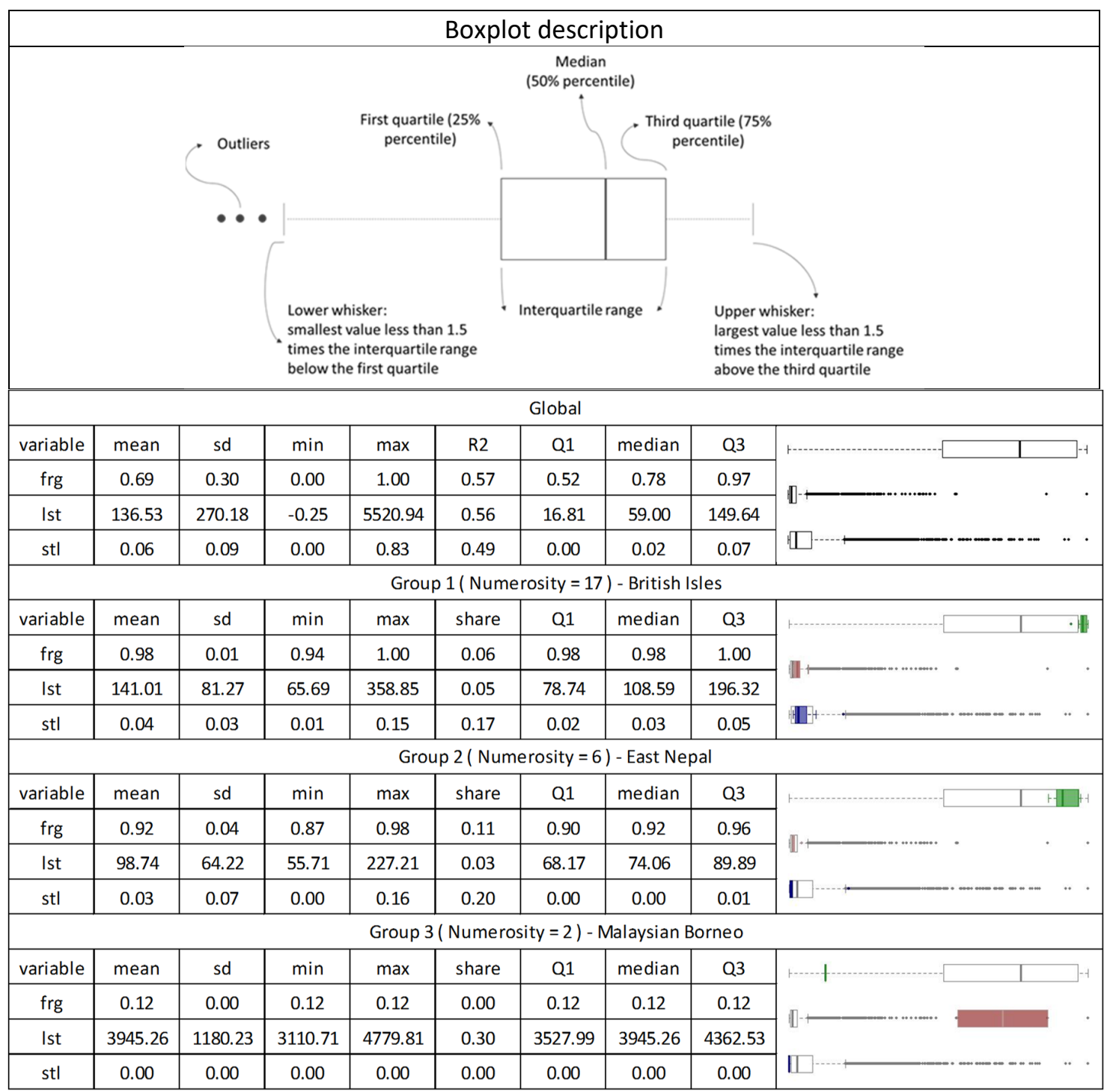


medRxiv preprint doi: https://doi.org/10.1101/2020.07.31.20166090; this version posted August 4, 2020. The copyright holder for this preprint (which was not certified by peer review) is the author/funder, who has granted medRxiv a license to display the preprint in

It is made available under a CC-BY-NC-ND 4.0 International license .

\begin{tabular}{|c|c|c|c|c|c|c|c|c|c|}
\hline \multicolumn{10}{|c|}{ Group 4 ( Numerosity $=9$ ) - East Bangladesh } \\
\hline variable & mean & sd & $\min$ & $\max$ & share & Q1 & median & Q3 & \multirow{4}{*}{ 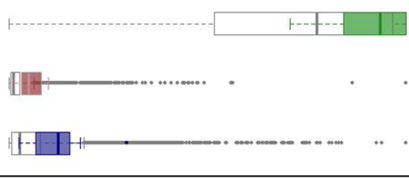 } \\
\hline $\mathrm{frg}$ & 0.91 & 0.10 & 0.71 & 1.00 & 0.29 & 0.84 & 0.94 & 1.00 & \\
\hline Ist & 296.63 & 170.07 & 74.13 & 544.77 & 0.09 & 166.44 & 272.83 & 447.55 & \\
\hline stl & 0.10 & 0.07 & 0.02 & 0.25 & 0.27 & 0.06 & 0.10 & 0.13 & \\
\hline \multicolumn{10}{|c|}{ Group 5 ( Numerosity = 34 ) - Southern Sichuan, Guizhou (China) } \\
\hline variable & mean & sd & $\min$ & $\max$ & share & Q1 & median & Q3 & \multirow{4}{*}{ 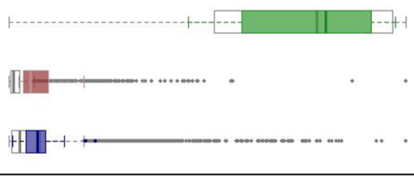 } \\
\hline frg & 0.75 & 0.18 & 0.45 & 0.97 & 0.52 & 0.59 & 0.80 & 0.91 & \\
\hline Ist & 398.06 & 247.72 & 134.08 & 1039.54 & 0.16 & 201.37 & 298.14 & 539.71 & \\
\hline stl & 0.06 & 0.04 & 0.01 & 0.18 & 0.21 & 0.04 & 0.06 & 0.08 & \\
\hline \multicolumn{10}{|c|}{ Group 6 ( Nume rosity = 188) - Iraq, Iran, Afghanistan } \\
\hline variable & mean & sd & $\min$ & $\max$ & share & Q1 & median & Q3 & \multirow{4}{*}{ 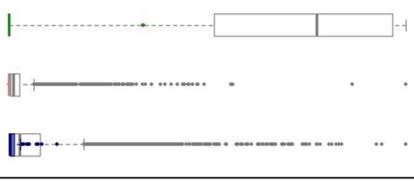 } \\
\hline frg & 0.00 & 0.03 & 0.00 & 0.34 & 0.34 & 0.00 & 0.00 & 0.00 & \\
\hline Ist & 0.05 & 0.35 & 0.00 & 3.08 & 0.00 & 0.00 & 0.00 & 0.00 & \\
\hline stl & 0.01 & 0.01 & 0.00 & 0.10 & 0.12 & 0.00 & 0.00 & 0.01 & \\
\hline \multicolumn{10}{|c|}{ Group $7($ Numerosity $=24)$ - East China } \\
\hline variable & mean & sd & $\min$ & $\max$ & share & Q1 & median & Q3 & \multirow{4}{*}{ 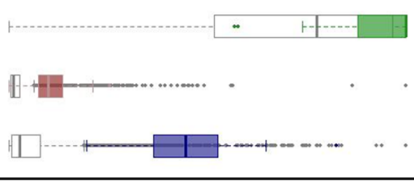 } \\
\hline $\mathrm{frg}$ & 0.92 & 0.13 & 0.57 & 1.00 & 0.43 & 0.89 & 1.00 & 1.00 & \\
\hline Ist & 603.50 & 307.95 & 0.00 & 1398.45 & 0.25 & 418.57 & 546.92 & 736.42 & \\
\hline stl & 0.37 & 0.12 & 0.16 & 0.68 & 0.63 & 0.31 & 0.37 & 0.43 & \\
\hline \multicolumn{10}{|c|}{ Group 8 ( Nume rosity = 185) - Japan, Shanghai to Hong Kong (China) } \\
\hline variable & mean & sd & $\min$ & $\max$ & share & Q1 & median & Q3 & \multirow{4}{*}{ 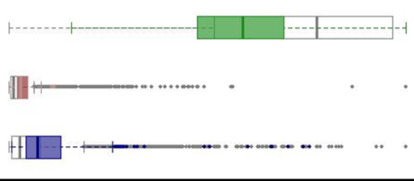 } \\
\hline $\mathrm{frg}$ & 0.57 & 0.18 & 0.16 & 1.00 & 0.84 & 0.47 & 0.59 & 0.69 & \\
\hline Ist & 189.25 & 108.77 & 16.37 & 631.17 & 0.11 & 110.50 & 163.29 & 257.94 & \\
\hline stl & 0.10 & 0.11 & 0.01 & 0.63 & 0.75 & 0.03 & 0.06 & 0.11 & \\
\hline \multicolumn{10}{|c|}{ Group 9 ( Nume rosity = 4) - Manila bay (Philippines) } \\
\hline variable & mean & sd & $\min$ & $\max$ & share & Q1 & median & $\mathrm{Q} 3$ & \multirow{4}{*}{ 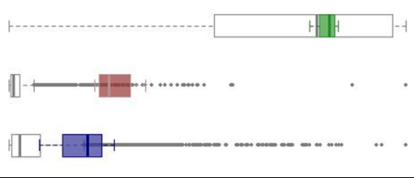 } \\
\hline frg & 0.80 & 0.03 & 0.76 & 0.83 & 0.07 & 0.79 & 0.81 & 0.81 & \\
\hline Ist & 1469.36 & 307.68 & 1193.82 & 1899.49 & 0.13 & 1286.61 & 1392.07 & 1574.83 & \\
\hline stl & 0.15 & 0.07 & 0.06 & 0.22 & 0.19 & 0.14 & 0.16 & 0.18 & \\
\hline \multicolumn{10}{|c|}{ Group 10 ( Numerosity = 13) - Central Sichuan (China) } \\
\hline variable & mean & $s d$ & $\min$ & $\max$ & share & Q1 & median & Q3 & \multirow{4}{*}{ 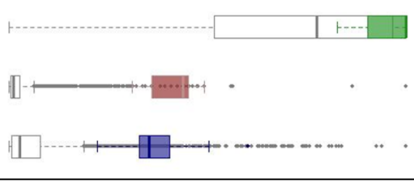 } \\
\hline frg & 0.96 & 0.06 & 0.83 & 1.00 & 0.17 & 0.90 & 1.00 & 1.00 & \\
\hline Ist & 2259.48 & 335.99 & 1713.00 & 2720.77 & 0.18 & 1986.94 & 2412.74 & 2489.97 & \\
\hline stl & 0.32 & 0.08 & 0.18 & 0.50 & 0.38 & 0.27 & 0.29 & 0.34 & \\
\hline \multicolumn{10}{|c|}{ Group 11 ( Numerosity = 118) - Northern Myanmar } \\
\hline variable & mean & sd & $\min$ & $\max$ & share & Q1 & median & Q3 & \multirow{4}{*}{ 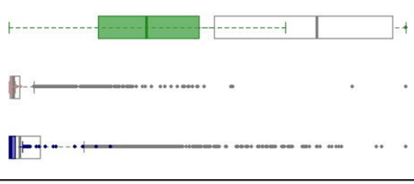 } \\
\hline frg & 0.35 & 0.18 & 0.00 & 1.00 & 1.00 & 0.23 & 0.35 & 0.48 & \\
\hline Ist & 26.58 & 23.05 & 0.13 & 152.78 & 0.03 & 9.94 & 19.75 & 38.37 & \\
\hline stl & 0.02 & 0.03 & 0.00 & 0.21 & 0.26 & 0.00 & 0.00 & 0.01 & \\
\hline \multicolumn{10}{|c|}{ Group 12 ( Numerosity = 18) - Lebanon, Syria } \\
\hline variable & mean & sd & $\min$ & $\max$ & share & Q1 & median & Q3 & \multirow{4}{*}{ 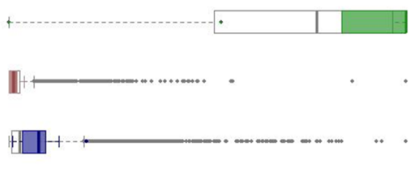 } \\
\hline $\mathrm{frg}$ & 0.80 & 0.38 & 0.00 & 1.00 & 1.00 & 0.88 & 1.00 & 1.00 & \\
\hline Ist & 50.82 & 76.73 & 0.00 & 204.68 & 0.04 & 0.00 & 2.57 & 88.93 & \\
\hline stl & 0.06 & 0.04 & 0.01 & 0.16 & 0.19 & 0.03 & 0.06 & 0.08 & \\
\hline
\end{tabular}


medRxiv preprint doi: https://doi.org/10.1101/2020.07.31.20166090; this version posted August 4, 2020. The copyright holder for this preprint (which was not certified by peer review) is the author/funder, who has granted medRxiv a license to display the preprint in

It is made available under a CC-BY-NC-ND 4.0 International license .

\begin{tabular}{|c|c|c|c|c|c|c|c|c|c|}
\hline \multicolumn{10}{|c|}{ Group 13 ( Numerosity = 41) - East Sichuan (China) } \\
\hline variable & mean & sd & $\min$ & $\max$ & share & Q1 & median & Q3 & \multirow{2}{*}{\begin{tabular}{|c|c|}
$\quad \cdots$ & $\ldots$
\end{tabular}} \\
\hline frg & 0.97 & 0.08 & 0.71 & 1.00 & 0.29 & 0.99 & 1.00 & 1.00 & \\
\hline Ist & 1207.95 & 196.18 & 865.09 & 1768.76 & 0.16 & 1063.81 & 1195.58 & 1315.51 & \multirow{2}{*}{$\mathbb{D}+\mathrm{H}+\cdots$} \\
\hline stl & 0.19 & 0.07 & 0.06 & 0.40 & 0.41 & 0.14 & 0.17 & 0.23 & \\
\hline \multicolumn{10}{|c|}{ Group 14 ( Numerosity = 732 ) - China } \\
\hline variable & mean & sd & $\min$ & $\max$ & share & Q1 & median & Q3 & \multirow{4}{*}{ 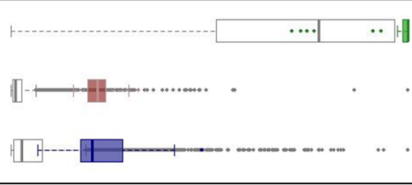 } \\
\hline frg & 0.85 & 0.14 & 0.29 & 1.00 & 0.71 & 0.77 & 0.89 & 0.97 & \\
\hline Ist & 260.33 & 188.99 & 63.54 & 1636.25 & 0.28 & 144.39 & 193.75 & 300.04 & \\
\hline stl & 0.11 & 0.09 & 0.00 & 0.64 & 0.77 & 0.04 & 0.08 & 0.14 & \\
\hline \multicolumn{10}{|c|}{ Group 15 ( Numerosity = 14) - West Assam, Sikkim, Bihar (India) } \\
\hline variable & mean & sd & $\min$ & $\max$ & share & Q1 & median & $\mathrm{Q} 3$ & \multirow{4}{*}{ D } \\
\hline frg & 0.83 & 0.29 & 0.00 & 1.00 & 1.00 & 0.86 & 0.97 & 0.99 & \\
\hline Ist & 306.08 & 69.22 & 186.08 & 442.16 & 0.05 & 279.78 & 298.44 & 335.38 & \\
\hline stl & 0.52 & 0.16 & 0.26 & 0.83 & 0.68 & 0.40 & 0.54 & 0.61 & \\
\hline \multicolumn{10}{|c|}{ Group 16 ( Numerosity $=27$ ) - Atlas Mountains } \\
\hline variable & mean & sd & $\min$ & $\max$ & share & Q1 & median & Q3 & \multirow{4}{*}{ 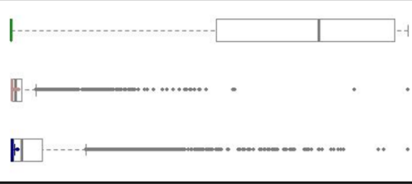 } \\
\hline frg & 0.00 & 0.00 & 0.00 & 0.00 & 0.00 & 0.00 & 0.00 & 0.00 & \\
\hline Ist & 20.45 & 26.97 & 3.17 & 103.64 & 0.02 & 4.84 & 8.10 & 19.57 & \\
\hline stl & 0.00 & 0.00 & 0.00 & 0.01 & 0.02 & 0.00 & 0.00 & 0.00 & \\
\hline \multicolumn{10}{|c|}{ Group 17 ( Numerosity = 14) - Assam (India) } \\
\hline variable & mean & sd & $\min$ & $\max$ & share & Q1 & median & Q3 & \multirow{4}{*}{ D } \\
\hline frg & 0.82 & 0.16 & 0.52 & 0.99 & 0.47 & 0.70 & 0.88 & 0.94 & \\
\hline Ist & 176.63 & 115.38 & 36.71 & 440.25 & 0.07 & 82.13 & 156.77 & 228.82 & \\
\hline stl & 0.47 & 0.16 & 0.27 & 0.77 & 0.60 & 0.31 & 0.48 & 0.59 & \\
\hline \multicolumn{10}{|c|}{ Group 18 ( Numerosity = 69) - Australia, South Papua } \\
\hline variable & mean & sd & $\min$ & $\max$ & share & Q1 & median & Q3 & \multirow{4}{*}{ 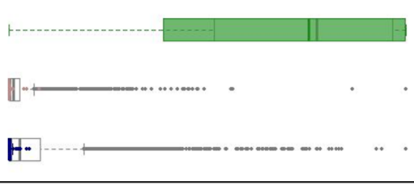 } \\
\hline frg & 0.69 & 0.29 & 0.00 & 1.00 & 1.00 & 0.39 & 0.76 & 1.00 & \\
\hline Ist & 18.32 & 62.87 & 0.00 & 419.57 & 0.08 & 0.00 & 0.65 & 9.82 & \\
\hline stl & 0.00 & 0.01 & 0.00 & 0.04 & 0.05 & 0.00 & 0.00 & 0.00 & \\
\hline \multicolumn{10}{|c|}{ Group 19 ( Numerosity = 84) - Philippines, Taiwan } \\
\hline variable & mean & sd & $\min$ & $\max$ & share & Q1 & median & Q3 & \multirow{4}{*}{ 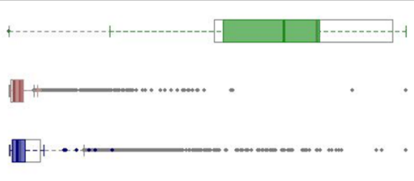 } \\
\hline frg & 0.63 & 0.23 & 0.00 & 1.00 & 1.00 & 0.54 & 0.69 & 0.78 & \\
\hline Ist & 135.73 & 107.16 & 10.45 & 427.88 & 0.08 & 51.91 & 103.28 & 189.72 & \\
\hline stl & 0.03 & 0.05 & 0.00 & 0.22 & 0.26 & 0.01 & 0.02 & 0.03 & \\
\hline \multicolumn{10}{|c|}{ Group 20 ( Numerosity = 294) - Borneo, Sulawesi, Papua } \\
\hline variable & mean & sd & $\min$ & $\max$ & share & Q1 & median & Q3 & \multirow{4}{*}{ 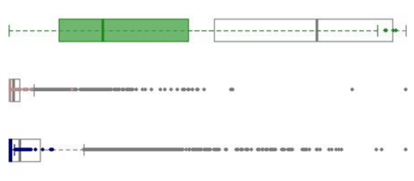 } \\
\hline frg & 0.32 & 0.24 & 0.00 & 0.98 & 0.98 & 0.13 & 0.24 & 0.45 & \\
\hline Ist & 20.76 & 66.09 & 0.00 & 877.45 & 0.16 & 1.85 & 4.46 & 11.72 & \\
\hline stl & 0.01 & 0.01 & 0.00 & 0.09 & 0.11 & 0.00 & 0.00 & 0.00 & \\
\hline \multicolumn{10}{|c|}{ Group 21 ( Numerosity = 39) India } \\
\hline variable & mean & sd & $\min$ & $\max$ & share & Q1 & median & Q3 & \multirow{4}{*}{ 南 } \\
\hline $\mathrm{frg}$ & 0.85 & 0.25 & 0.00 & 1.00 & 1.00 & 0.73 & 1.00 & 1.00 & \\
\hline Ist & 69.90 & 42.61 & 12.35 & 239.52 & 0.04 & 41.12 & 66.94 & 85.47 & \\
\hline stl & 0.13 & 0.14 & 0.00 & 0.53 & 0.64 & 0.00 & 0.10 & 0.24 & \\
\hline
\end{tabular}


medRxiv preprint doi: https://doi.org/10.1101/2020.07.31.20166090; this version posted August 4, 2020. The copyright holder for this preprint (which was not certified by peer review) is the author/funder, who has granted medRxiv a license to display the preprint in

It is made available under a CC-BY-NC-ND 4.0 International license .

\begin{tabular}{|c|c|c|c|c|c|c|c|c|c|}
\hline \multicolumn{10}{|c|}{ Group 22 ( Numerosity = 320) - Italy, Central Europe, Balkans } \\
\hline variable & mean & sd & $\min$ & $\max$ & share & Q1 & median & Q3 & \multirow{2}{*}{ +1 } \\
\hline frg & 0.73 & 0.16 & 0.38 & 1.00 & 0.62 & 0.60 & 0.73 & 0.84 & \\
\hline Ist & 81.91 & 74.65 & 4.53 & 695.83 & 0.13 & 37.22 & 73.12 & 105.16 & \multirow{2}{*}{ 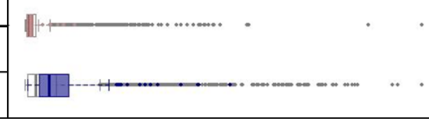 } \\
\hline stl & 0.07 & 0.06 & 0.00 & 0.43 & 0.51 & 0.03 & 0.05 & 0.09 & \\
\hline \multicolumn{10}{|c|}{ Group 23 ( Numerosity = 26) - Saudi Arabia } \\
\hline variable & mean & sd & $\min$ & $\max$ & share & Q1 & median & Q3 & \multirow{4}{*}{ 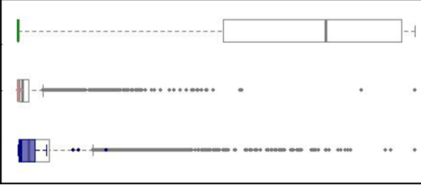 } \\
\hline frg & 0.00 & 0.00 & 0.00 & 0.00 & 0.00 & 0.00 & 0.00 & 0.00 & \\
\hline Ist & 2.09 & 8.76 & -0.25 & 43.76 & 0.01 & 0.00 & 0.00 & 0.00 & \\
\hline stl & 0.03 & 0.05 & 0.00 & 0.18 & 0.22 & 0.00 & 0.01 & 0.03 & \\
\hline \multicolumn{10}{|c|}{ Group 24 ( Numerosity = 1) - Shijiazhuang (Hebei, China) } \\
\hline variable & mean & sd & $\min$ & $\max$ & share & Q1 & median & Q3 & \multirow{4}{*}{ 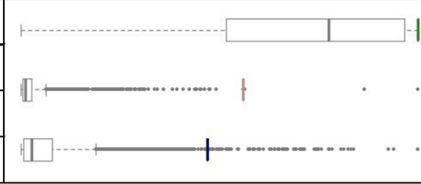 } \\
\hline frg & 1.00 & NA & 1.00 & 1.00 & 0.00 & 1.00 & 1.00 & 1.00 & \\
\hline Ist & 3084.02 & NA & 3084.02 & 3084.02 & 0.00 & 3084.02 & 3084.02 & 3084.02 & \\
\hline stl & 0.39 & NA & 0.39 & 0.39 & 0.00 & 0.39 & 0.39 & 0.39 & \\
\hline \multicolumn{10}{|c|}{ Group 25 ( Numerosity = 1202 ) - Southeast Asia, Sumatra, Java } \\
\hline variable & mean & sd & $\min$ & $\max$ & share & Q1 & median & Q3 & \multirow{4}{*}{ 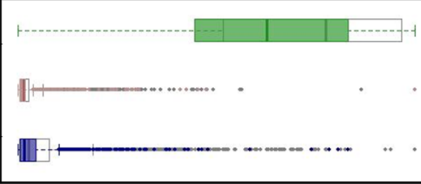 } \\
\hline frg & 0.63 & 0.24 & 0.00 & 1.00 & 1.00 & 0.45 & 0.63 & 0.83 & \\
\hline Ist & 118.12 & 287.24 & 0.00 & 5520.94 & 1.00 & 21.93 & 45.12 & 95.65 & \\
\hline stl & 0.03 & 0.07 & 0.00 & 0.69 & 0.83 & 0.00 & 0.01 & 0.04 & \\
\hline \multicolumn{10}{|c|}{ Group 26 ( Numerosity = 283) - Easy Myanmar, South Yunnan (China) } \\
\hline variable & mean & sd & $\min$ & $\max$ & share & Q1 & median & Q3 & \multirow{4}{*}{ 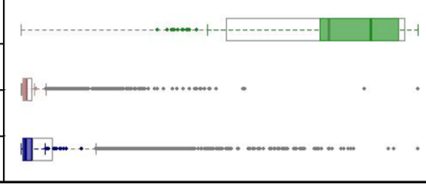 } \\
\hline frg & 0.83 & 0.16 & 0.34 & 1.00 & 0.66 & 0.75 & 0.88 & 0.95 & \\
\hline Ist & 60.25 & 46.74 & 3.56 & 211.33 & 0.04 & 22.65 & 50.62 & 85.02 & \\
\hline stl & 0.02 & 0.02 & 0.00 & 0.13 & 0.15 & 0.00 & 0.01 & 0.02 & \\
\hline \multicolumn{10}{|c|}{ Group 27 ( Numerosity = 18) - Henan, Shandong (China) } \\
\hline variable & mean & sd & $\min$ & $\max$ & share & Q1 & median & Q3 & \multirow{4}{*}{ 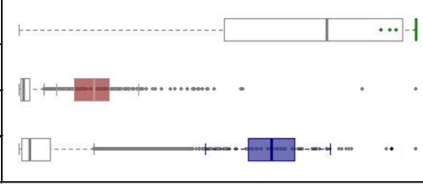 } \\
\hline frg & 0.99 & 0.03 & 0.91 & 1.00 & 0.09 & 1.00 & 1.00 & 1.00 & \\
\hline Ist & 1030.48 & 298.09 & 519.25 & 1664.13 & 0.21 & 782.27 & 1045.74 & 1210.37 & \\
\hline stl & 0.54 & 0.10 & 0.39 & 0.78 & 0.47 & 0.48 & 0.53 & 0.57 & \\
\hline \multicolumn{10}{|c|}{ Group 28 ( Numerosity = 716) - Eastern Europe, Asia, West Myanmar } \\
\hline variable & mean & sd & $\min$ & $\max$ & share & Q1 & median & Q3 & \multirow{4}{*}{ 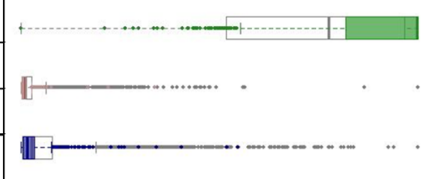 } \\
\hline frg & 0.87 & 0.23 & 0.00 & 1.00 & 1.00 & 0.82 & 1.00 & 1.00 & \\
\hline Ist & 49.40 & 105.81 & 0.00 & 1861.40 & 0.34 & 3.04 & 22.39 & 60.20 & \\
\hline stl & 0.02 & 0.04 & 0.00 & 0.45 & 0.55 & 0.00 & 0.01 & 0.03 & \\
\hline \multicolumn{10}{|c|}{ Group 29( Numerosity = 153) - Yunnan, Central to Northeast China } \\
\hline variable & mean & sd & $\min$ & $\max$ & share & Q1 & median & Q3 & \multirow{4}{*}{ 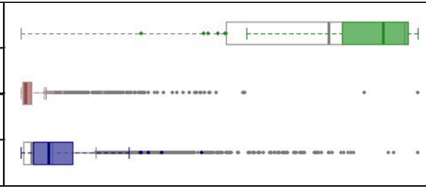 } \\
\hline frg & 0.87 & 0.14 & 0.30 & 1.00 & 0.70 & 0.81 & 0.91 & 0.98 & \\
\hline Ist & 122.43 & 135.54 & 1.24 & 1137.94 & 0.21 & 35.82 & 104.68 & 147.99 & \\
\hline stl & 0.08 & 0.07 & 0.00 & 0.38 & 0.46 & 0.03 & 0.06 & 0.11 & \\
\hline \multicolumn{10}{|c|}{ Group 30 ( Nume rosity = 349) - Western Europe, Maghreb } \\
\hline variable & mean & sd & $\min$ & $\max$ & share & Q1 & median & Q3 & \multirow{4}{*}{ 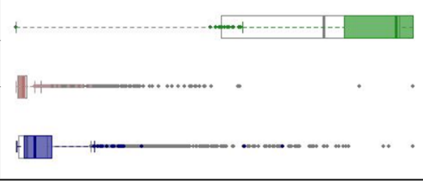 } \\
\hline frg & 0.88 & 0.16 & 0.00 & 1.00 & 1.00 & 0.83 & 0.96 & 1.00 & \\
\hline Ist & 105.59 & 132.63 & 2.48 & 1040.61 & 0.19 & 34.59 & 64.49 & 125.01 & \\
\hline stl & 0.06 & 0.06 & 0.00 & 0.56 & 0.67 & 0.02 & 0.04 & 0.07 & \\
\hline
\end{tabular}


medRxiv preprint doi: https://doi.org/10.1101/2020.07.31.20166090; this version posted August 4, 2020. The copyright holder for this preprint (which was not certified by peer review) is the author/funder, who has granted medRxiv a license to display the preprint in It is made available under a CC-BY-NC-ND 4.0 International license.
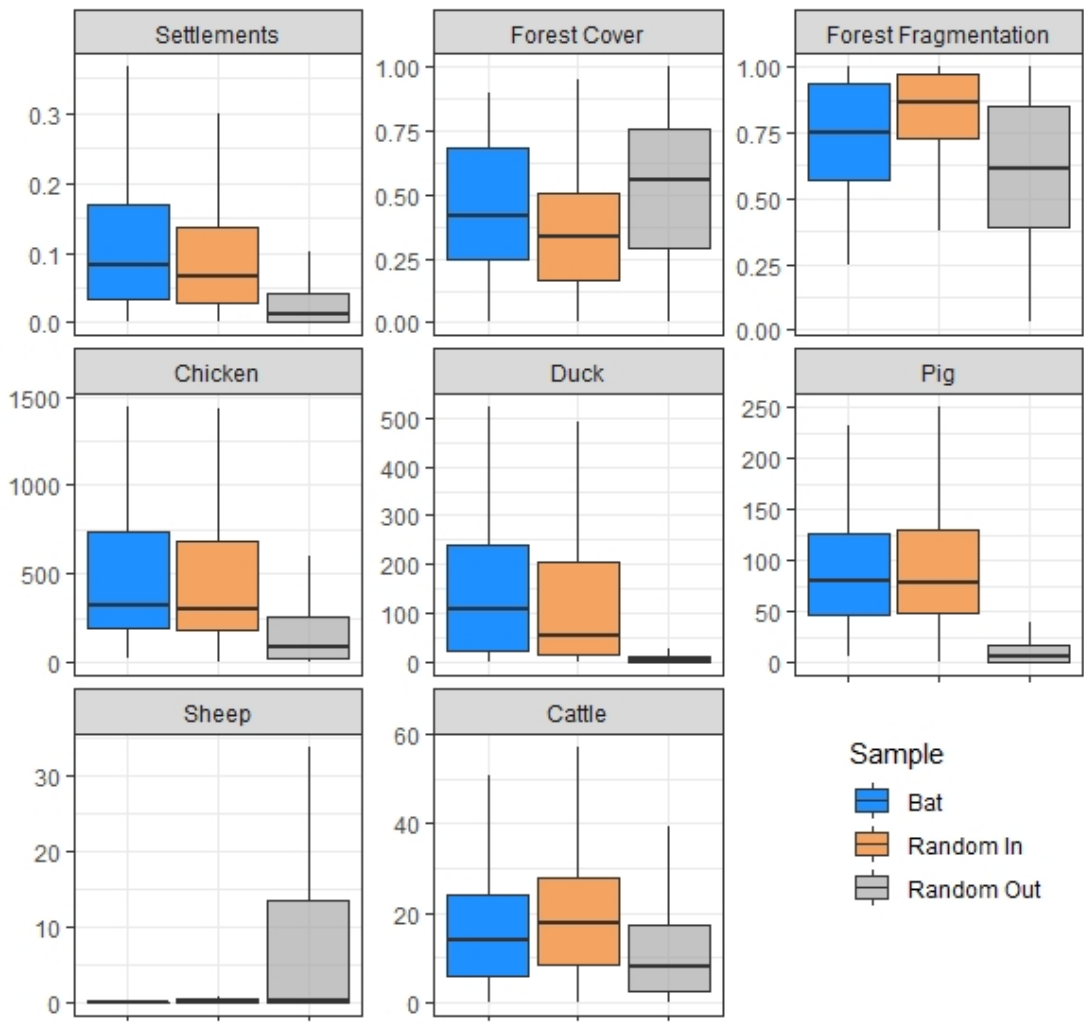

Figure S1. Boxplots of the distribution data of indicators. In blue are reported data in bat locations, in orange data in China, in grey data outside China. 
medRxiv preprint doi: https://doi.org/10.1101/2020.07.31.20166090; this version posted August 4, 2020. The copyright holder for this preprint (which was not certified by peer review) is the author/funder, who has granted medRxiv a license to display the preprint in It is made available under a CC-BY-NC-ND 4.0 International license.
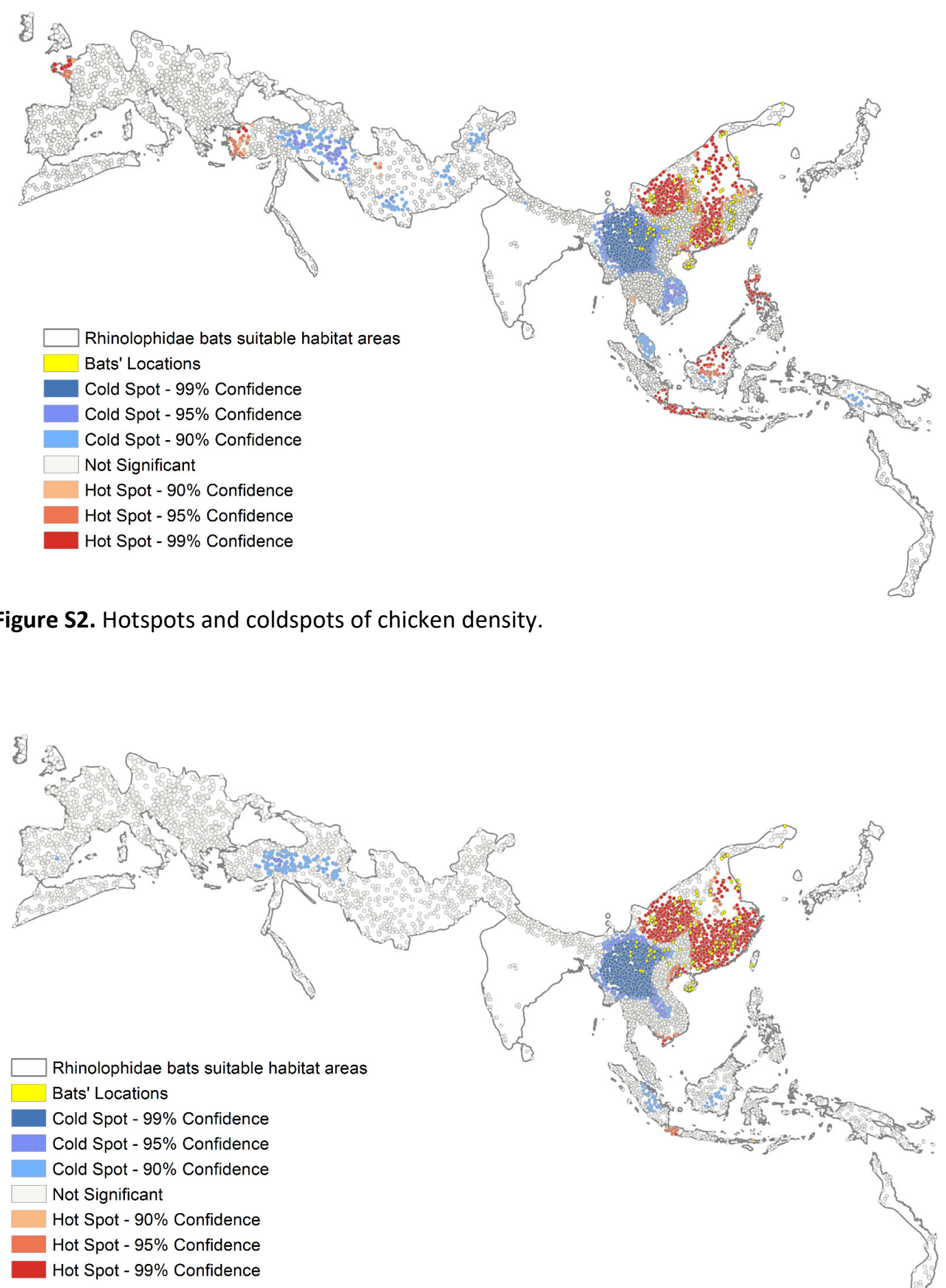

Figure S2. Hotspots and coldspots of chicken density.

Figure S3. Hotspots and coldspots of duck density. 
medRxiv preprint doi: https://doi.org/10.1101/2020.07.31.20166090; this version posted August 4, 2020. The copyright holder for this preprint (which was not certified by peer review) is the author/funder, who has granted medRxiv a license to display the preprint in It is made available under a CC-BY-NC-ND 4.0 International license.

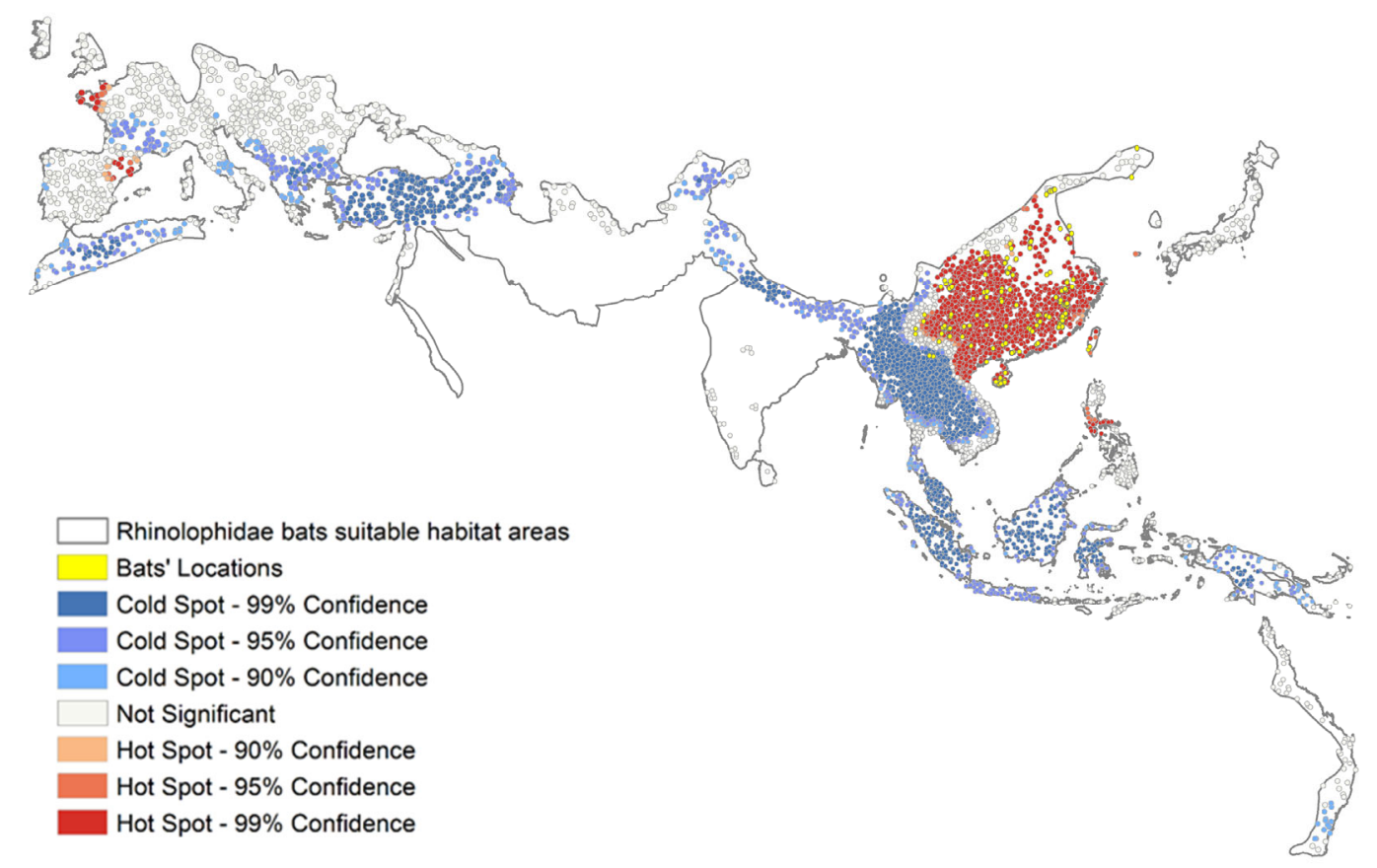

591

592 Figure S4. Hotspots and coldspots of pig density.

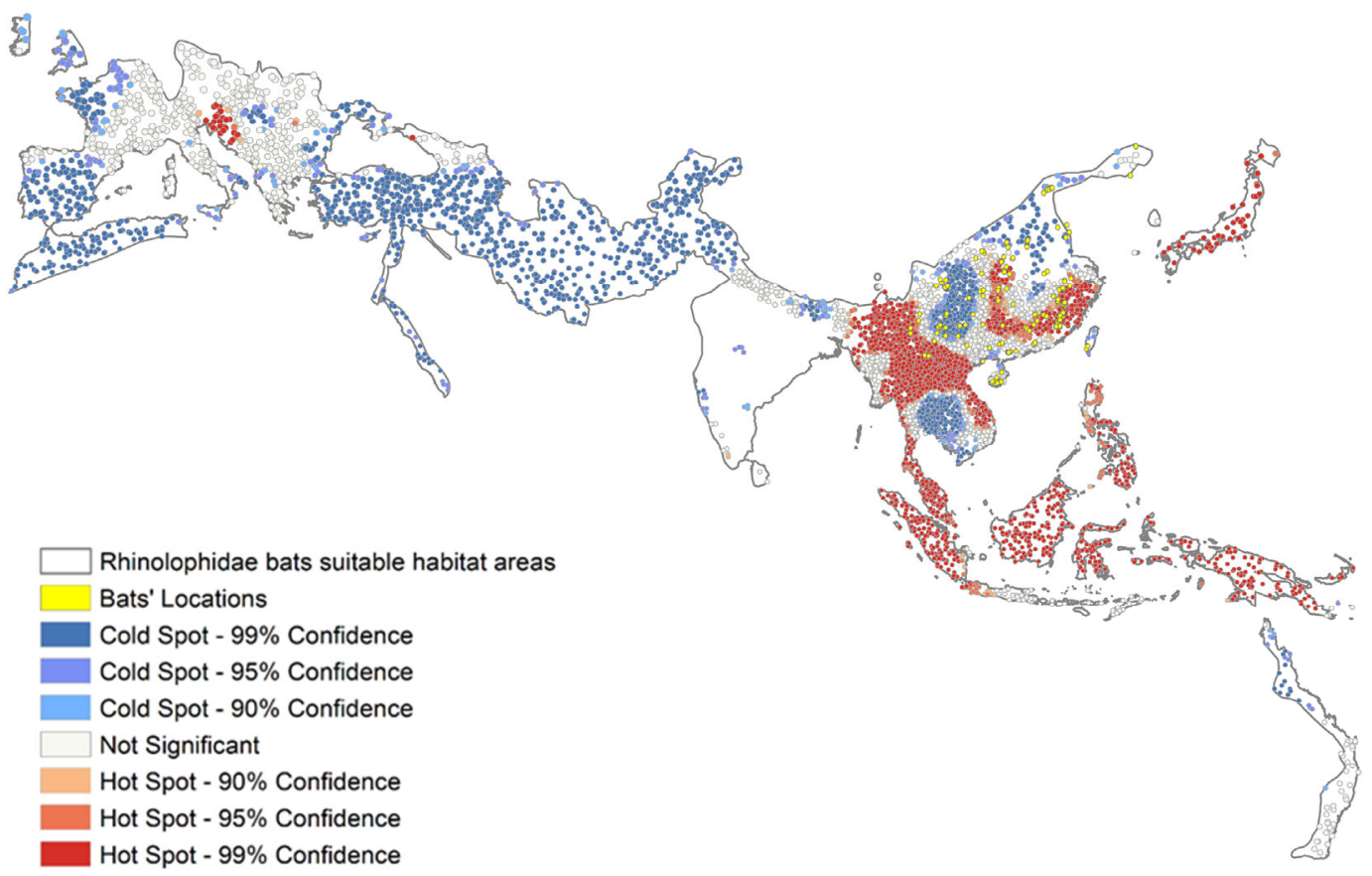

594 Figure S5. Hotspots and coldspots of forest cover 
medRxiv preprint doi: https://doi.org/10.1101/2020.07.31.20166090; this version posted August 4, 2020. The copyright holder for this preprint (which was not certified by peer review) is the author/funder, who has granted medRxiv a license to display the preprint in

It is made available under a CC-BY-NC-ND 4.0 International license .

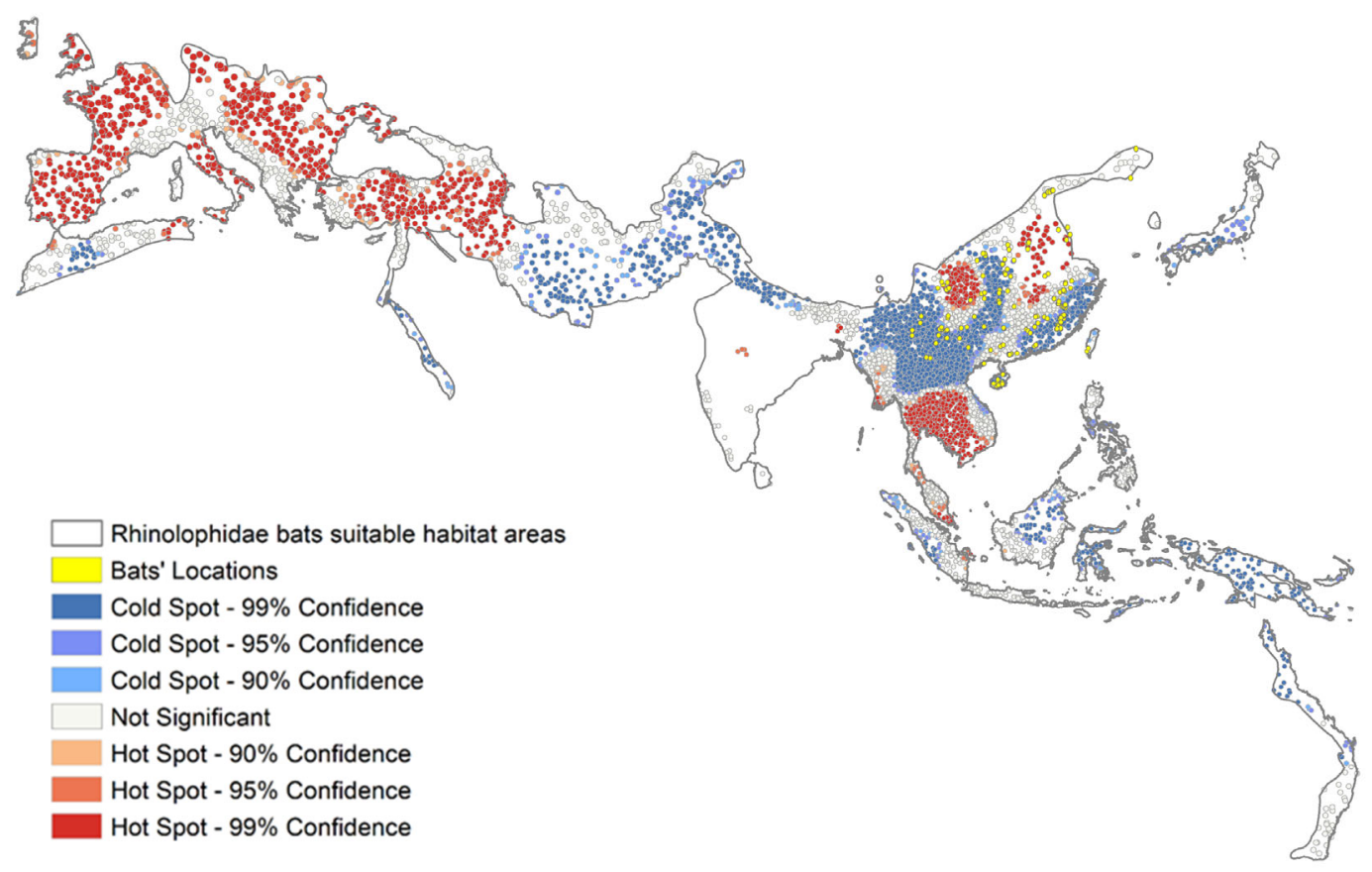

595

$596 \quad$ Figure S6. Hotspots and coldspots of cropland cover.

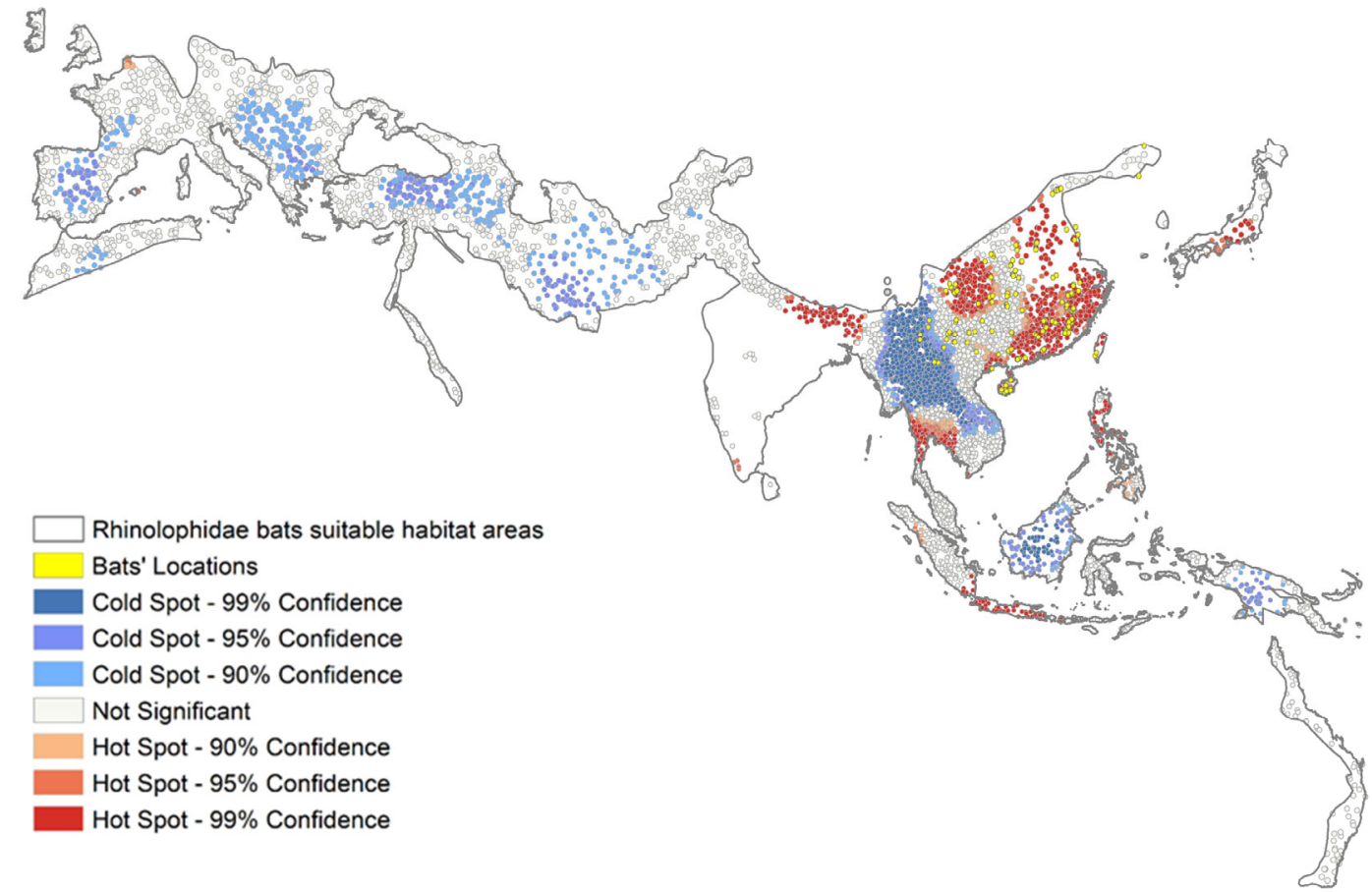

598 Figure S7. Hotspots and coldspots of population density. 
medRxiv preprint doi: https://doi.org/10.1101/2020.07.31.20166090; this version posted August 4, 2020. The copyright holder for this preprint (which was not certified by peer review) is the author/funder, who has granted medRxiv a license to display the preprint in It is made available under a CC-BY-NC-ND 4.0 International license.
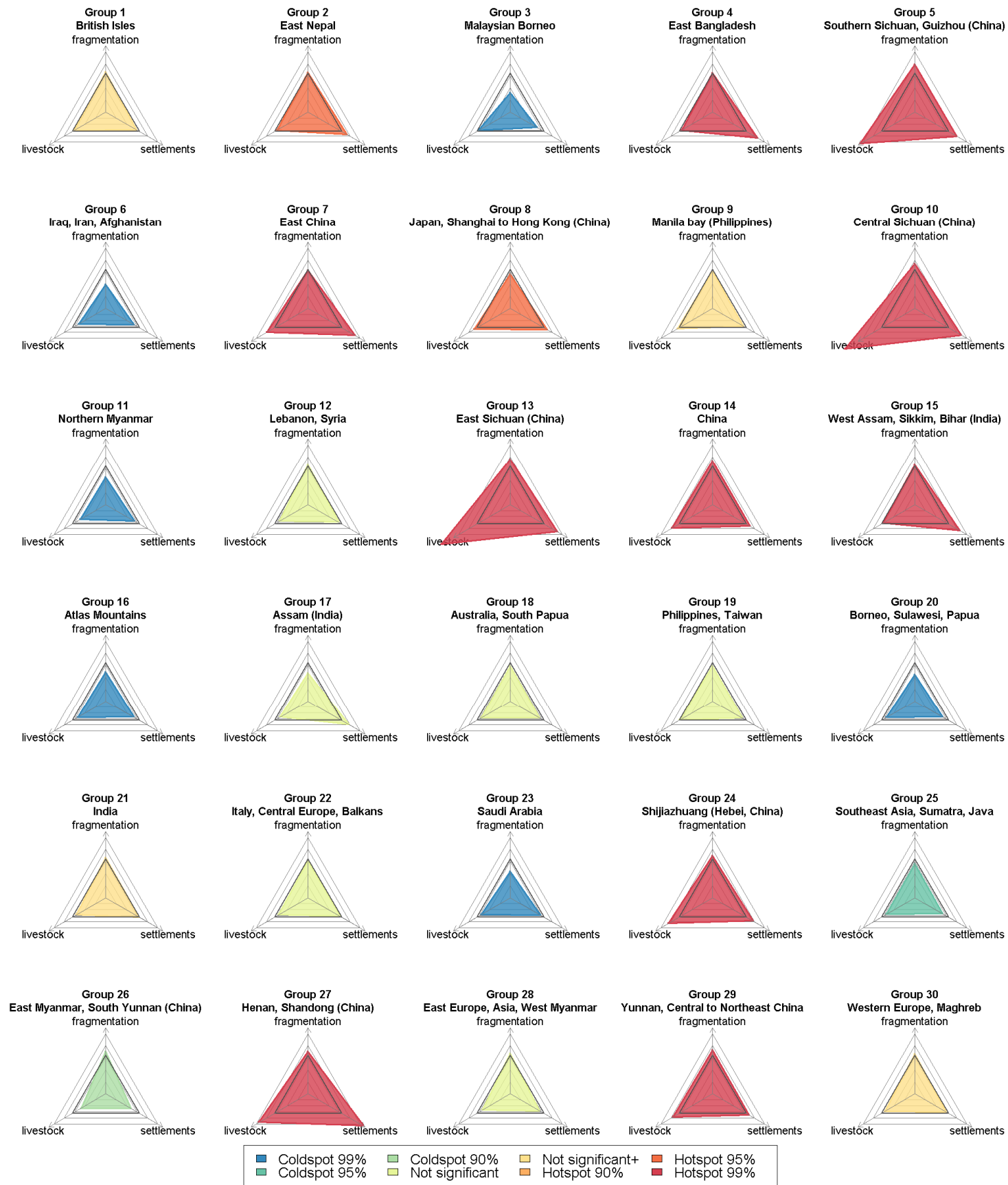

600 Figure S8. Group analysis. Trajectories of risk. 